\title{
Second-order water wave diffraction by an array of vertical cylinders
}

\author{
By Š. MALENICA ${ }^{1}$, R. EATOCK TAYLOR \\ AND J. B. H U A N G \\ ${ }^{1}$ Bureau Veritas, 17 bis Place des Reflets, La Défense 2 FR 92400, Courbevoie, France \\ ${ }^{2}$ Department of Engineering Science, University of Oxford, OX1 3PJ, UK
}

(Received 26 June 1998 and in revised form 29 January 1999)

The problem of second-order water wave diffraction of an incident monochromatic wave field by an array of bottom-mounted circular cylinders is solved by a semianalytical approach. The solution for the second-order potential is obtained by combining eigenfunction expansions with an integral representation. Unlike the indirect approach for second-order forces (Lighthill 1979; Molin 1979), this approach gives complete information about local flow characteristics (pressure, velocities, wave elevation, etc.) thus providing a basis for solving the third-order problem. The results obtained are compared with other published data, and new detailed results, useful for benchmarking purposes, are given. Finally the influences of wave incidence, cylinder radius and cylinder configuration are considered. This leads to the suggestion that there exists a near-trapping phenomenon for the second-order wave in an array of cylinders, at half the wave frequency at which the corresponding linear near-trapped mode occurs.

\section{Introduction}

Even under the simplifying assumptions of potential flow, the nonlinear interaction of water waves with floating bodies is too difficult a problem to be treated directly. Only a few studies intended to solve the complete three-dimensional problem in the time domain are known (e.g. Romate 1989; Ferrant 1996; Xue \& Yue 1995). Some encouraging results for simple configurations have been obtained, though the computational times for these kinds of calculations are still prohibitive in a design context. There is still a need for simple approaches which capture the essential features of the hydrodynamics.

Linearization is the usual way to treat the wave-body interaction. This leads to a relatively much simpler problem and one can reasonably state that the analysis of linearized water wave diffraction-radiation by arbitrary floating bodies (not having forward speed) is now completely mastered. The linear solution however has several limitations. Perhaps the most important is its inability to predict the loads at frequencies different from those contained in the incident wave spectrum. It is known from physical experiments that responses of a floating body can occur at its natural frequencies, at which however the incident wave spectrum may have negligible energy. The observations indicate that the responses at these frequencies can be very significant. The excitation at these frequencies, however, can be explained only by introducing nonlinearities into the model. This is why 'second-order hydrodynamics' has received much attention in the recent past. It is now generally accepted that 
second-order wave loads are the main source of excitation for the slow drift motions of slack moored floating structures; and for the high-frequency resonant motion ('springing') of tension leg platforms (TLPs). Molin (1994) gives a review of the latter problem. These second-order loads can be formulated by an elegant indirect approach, based on the use of the Haskind relations (Lighthill 1979; Molin 1979), which avoids explicit calculation of the second-order potential. Numerical codes exist today which calculate the springing loads by means of this approach (Chen, Molin \& Petitjean 1991; Newman \& Lee 1992; Eatock Taylor \& Chau 1992). Another method of obtaining the second-order forces is the direct integration of the pressure over the body surface up to second order (e.g. Chau 1989; Kim \& Yue 1989; Scolan \& Molin 1989; Kriebel 1990). Compared with the indirect one, this method is more complex to implement, but it has the benefit of giving detailed information about the local features of the flow (pressure, particle velocities, wave elevation, etc).

The direct method was used by Chau \& Eatock Taylor (1992) to formulate the second-order diffraction problem for a single bottom-mounted circular cylinder. The authors used a special kind of Green function which, in addition to the homogeneous free surface condition, also satisfies the condition of zero normal velocity on the cylinder surface. The resulting expression for the second-order potential was reproduced by Malenica \& Molin (1995), using the ordinary wave Green function method, and by Newman (1996) using the Weber transform. By this approach, the expression for the second-order potential at any point in the fluid is obtained in the form of an eigenfunction expansion, with the coefficients accounting for the integration of the forcing term on the free surface. This approach can also be extended to truncated cylinders, and to the case of bichromatic waves (Huang \& Eatock Taylor 1996a; Eatock Taylor \& Huang 1997a,b). In the case of single bodies, the troublesome integral over the free surface can be evaluated efficiently so that even the thirdorder problem can be treated (as shown by Malenica \& Molin 1995 for a single bottom-seated cylinder). For practical purposes, however, it is necessary to analyse multi-column structures such as TLPs; but unfortunately these may not be modelled reliably as groups of isolated columns. This is because interaction effects between the columns are found to be very important at first and second order (Chau 1989; Linton \& Evans 1990; Chen 1991; Evans \& Porter 1997; Scolan \& Malenica 1998), and can also be anticipated to have a profound influence on behaviour at higher orders.

The third-order problem becomes important in view of the so-called 'ringing' phenomenon, which has been observed on some deep water offshore structures such as TLPs and gravity base structures (GBSs). Ringing appears at frequencies 3 to 5 times the peak frequency of the incoming wave system so that second-order theory cannot provide an explanation for the phenomenon: a higher-order nonlinear formulation is required. In order to tackle the third-order problem, the second-order problem must first be completely mastered so that accurate evaluation of the second-order potential and its derivatives on the free surface becomes possible. Since the numerical methods for calculating the second-order potential are very expensive, and in some cases not sufficiently precise, a semi-analytical approach for idealized multi-column configurations could be very useful. The present paper pursues this idea, following an approach similar to that initiated independently by Huang \& Eatock Taylor (1996b) and Malenica (1997).

The arrangement of the paper is as follows. Section 2 recapitulates the general formulation for second-order wave diffraction, and $\S 3$ describes the development of the solutions for the first- and second-order potentials. The latter is divided into 
different components, and careful attention is given to that part which is defined to satisfy an inhomogeneous condition on the free surface and a homogeneous condition on the surface of the cylinders. Section 4 discusses details of the numerical implementation, and this is followed by results which demonstrate convergence of the series and illustrate features of the second-order interaction phenomena arising with multiple cylinders.

\section{General formulation}

\subsection{The boundary value problem}

We adopt the usual framework of potential flow theory, assuming that the fluid is incompressible and irrotational, so that the governing equation in the fluid becomes Laplace's equation for the velocity potential $\Phi(x, y, z, t)$. We use Cartesian coordinates $\boldsymbol{x}=(x, y, z)$, with $x, y$ lying in the undisturbed free surface and $z$ pointing vertically upwards. The velocity field is the gradient of the potential, $\boldsymbol{v}=\nabla \Phi$, and pressure is calculated from the Bernoulli equation. The solution is assumed to be periodic in time, so that the time dependence can be factorized. In the bichromatic wave case, this means that the second order effects will appear at the frequencies $\left(\omega_{i} \pm \omega_{j}\right)$ where $\omega_{i}$ and $\omega_{j}$ are the frequencies of each wave component. The monochromatic wave field treated here can be regarded as a special case in which $\omega_{i}=\omega_{j}=\omega$. Thus, if we assume the following perturbation series for the potential:

$$
\left.\Phi=\epsilon \phi^{(1)}(\boldsymbol{x}, t)+\epsilon^{2} \phi^{(2)} \boldsymbol{x}, t\right)+\cdots,
$$

the first- and second-order potentials $\phi^{(1)}$ and $\phi^{(2)}$ (and other quantities such as pressure or wave kinematics) will have the following form:

$$
\begin{gathered}
\epsilon \phi^{(1)}(\boldsymbol{x}, t)=\operatorname{Re}\left\{\varphi^{(1)}(\boldsymbol{x}) \mathrm{e}^{-\mathrm{i} \omega t}\right\}, \\
\epsilon^{2} \phi^{(2)}(\boldsymbol{x}, t)=\bar{\varphi}^{(2)}(\boldsymbol{x})+\operatorname{Re}\left\{\varphi^{(2)}(\boldsymbol{x}) \mathrm{e}^{-2 \mathrm{i} \omega t}\right\} .
\end{gathered}
$$

After introducing the perturbation series, equation (2.1), into the original nonlinear boundary value problem (BVP) we can obtain corresponding BVPs for the potentials at the different orders. The details of the derivation are well known and will not be repeated here. We just recall the final expressions for the free surface conditions which cause the main difficulty in the resolution of problems of this type (here we are interested only in the periodic components, so that the potential $\bar{\varphi}^{(2)}$ will not be treated). Thus at first and second order the conditions are

$$
\begin{gathered}
-v \varphi^{(1)}+\frac{\partial \varphi^{(1)}}{\partial z}=0 \\
-4 v \varphi^{(2)}+\frac{\partial \varphi^{(2)}}{\partial z}=\frac{\mathrm{i} \omega}{g}\left[\nabla \varphi^{(1)} \nabla \varphi^{(1)}-\frac{1}{2} \varphi^{(1)}\left(\frac{\partial^{2} \varphi^{(1)}}{\partial z^{2}}-v \frac{\partial \varphi^{(1)}}{\partial z}\right)\right],
\end{gathered}
$$

where $v=\omega^{2} / g$ is the infinite-depth wavenumber, $g$ is gravity, and the expressions are to be evaluated for $z=0$. In addition, the potential must satisfy the condition of no flow on the fixed boundaries, and the radiation condition at infinity.

\subsection{Expressions for wave elevation, pressure and forces}

The expressions for the free surface elevation components at first and second order are

$$
\eta^{(1)}=\frac{i \omega}{g} \varphi^{(1)},
$$




$$
\eta^{(2)}=\frac{2 \mathrm{i} \omega}{g} \varphi^{(2)}-\frac{1}{4 g} \nabla \varphi^{(1)} \nabla \varphi^{(1)}-\frac{v^{2}}{2 g} \varphi^{(1)} \varphi^{(1)} .
$$

After introducing the perturbation series, equation (2.1), into the Bernoulli equation

$$
p=-\varrho g z-\varrho \frac{\partial \Phi}{\partial t}-\frac{1}{2} \varrho \nabla \Phi \nabla \Phi,
$$

the dynamic pressures at the different orders are obtained as

$$
\begin{gathered}
p^{(1)}=\mathrm{i} \omega \varrho \varphi^{(1)}, \\
p^{(2)}=2 \mathrm{i} \omega \varrho \varphi^{(2)}-\frac{1}{4} \varrho \nabla \varphi^{(1)} \nabla \varphi^{(1)},
\end{gathered}
$$

where $\varrho$ is the density of the fluid.

The loads are calculated by integrating pressure over the wetted body surface. We obtain the following expressions for the components of surge force and pitch moment (about the $y$-axis):

$$
\begin{gathered}
F_{x}^{(1)}=\iint_{S_{B 0}} \mathrm{i} \omega \varrho \varphi^{(1)} n_{x} \mathrm{~d} S, \\
F_{x}^{(2)}=\iint_{S_{B 0}}\left(2 \mathrm{i} \omega \varrho \varphi^{(2)}-\frac{1}{4} \varrho \nabla \varphi^{(1)} \nabla \varphi^{(1)}\right) n_{x} \mathrm{~d} S+\frac{1}{4} \varrho g \int_{C_{B 0}} \eta^{(1)} \eta^{(1)} n_{x} \mathrm{~d} C, \\
M_{y}^{(1)}=\iint_{S_{B 0}} \mathrm{i} \omega \varrho \varphi^{(1)} z n_{x} \mathrm{~d} S, \\
M_{y}^{(2)}=\iint_{S_{B 0}}\left(2 \mathrm{i} \omega \varrho \varphi^{(2)}-\frac{1}{4} \varrho \nabla \varphi^{(1)} \nabla \varphi^{(1)}\right) z n_{x} \mathrm{~d} S+\frac{1}{4} \varrho g \int_{C_{B 0}} \eta^{(1)} \eta^{(1)} z n_{x} \mathrm{~d} C,
\end{gathered}
$$

where $S_{B 0}$ is the mean wetted surface of the body, $C_{B 0}$ its waterline and $n_{x}$ is the $x$-component of the normal (chosen to point out of the fluid domain). We are here assuming that the body is composed of vertical cylinders reaching to the seabed, so the only contribution to the moment is from the vertical surfaces.)

\section{Solution for the potentials}

At both first and second order, the potential $\varphi$ is further decomposed into incident and diffracted wave parts $\left(\varphi=\varphi_{I}+\varphi_{D}\right)$. Each of the potentials is treated separately.

\subsection{Incident potential}

The first and second components of the incident potential can be written in the forms

$$
\begin{gathered}
\varphi_{I}^{(1)}=-\frac{\mathrm{i} g A}{\omega} f_{0}^{(1)}(z) \mathrm{e}^{\mathrm{i} k_{0}(x \cos \beta+y \sin \beta)} \\
=-\frac{\mathrm{i} g A}{\omega} f_{0}^{(1)}(z) \sum_{m=-\infty}^{\infty} \mathrm{e}^{\mathrm{i} m(\pi / 2-\beta)} J_{m}\left(k_{0} r\right) \mathrm{e}^{\mathrm{i} m \theta}, \\
\varphi_{I}^{(2)}=\frac{3 \mathrm{i} \omega v A^{2}}{2 \sinh ^{2} k_{0} h} f_{I}^{(2)}(z) \mathrm{e}^{2 \mathrm{i} k_{0}(x \cos \beta+y \sin \beta)} \\
=\frac{3 \mathrm{i} \omega v A^{2}}{2 \sinh ^{2} k_{0} h} f_{I}^{(2)}(z) \sum_{m=-\infty}^{\infty} \mathrm{e}^{\mathrm{i} m(\pi / 2-\beta)} J_{m}\left(2 k_{0} r\right) \mathrm{e}^{\mathrm{i} m \theta},
\end{gathered}
$$


where $A$ is the amplitude of the incident wave, $\beta$ is the incident angle relative to the $x$-axis, $h$ is the water depth, $k_{0}$ is the finite-depth wavenumber $\left(k_{0} \tanh k_{0} h=v\right)$, and $J_{m}$ denotes the $m$ th-order Bessel function. Furthermore

$$
f_{0}^{(1)}(z)=\frac{\cosh k_{0}(z+h)}{\cosh k_{0} h}, \quad f_{I}^{(2)}(z)=-\frac{\cosh 2 k_{0}(z+h)}{4 v \sinh ^{2} k_{0} h} .
$$

\subsection{First-order diffraction potential}

All notation corresponds to the multiple cylinder configuration presented in figure 1; $a_{k}$ is the radius of the $k$ th cylinder, of which there are $N_{c}$. The first-order diffraction solution used here was given by Linton \& Evans (1990). We just recall the basic steps. The diffraction potential $\varphi_{D}^{(1)}$ is first assumed to have the general form

$$
\varphi_{D}^{(1)}=f_{0}^{(1)}(z) \sum_{k=1}^{N_{c}} \sum_{m=-\infty}^{\infty} A_{m}^{k} Z_{m 0}^{(1) k} H_{m}\left(k_{0} r_{k}\right) \mathrm{e}^{\mathrm{i} m \theta_{k}},
$$

where $Z_{m 0}^{(1) k}=J_{m}^{\prime}\left(k_{0} a_{k}\right) / H_{m}^{\prime}\left(k_{0} a_{k}\right)$, and $H_{m}$ stands for the Hankel function $H_{m}=$ $J_{m}+\mathrm{i} Y_{m}$. The use of Graf's addition theorem for Bessel functions (Abramowitz \& Stegun 1970) facilitates the application of the boundary conditions on each cylinder $\left(\partial \varphi_{D}^{(1)} / \partial n=-\partial \varphi_{I}^{(1)} / \partial n\right)$, so that the interaction coefficients $A_{m}^{k}$ can be found as solutions of the linear system of equations

$$
A_{m}^{k}+\sum_{j \neq k}^{N_{c}} \sum_{n=-\infty}^{\infty} A_{n}^{j} Z_{n 0}^{(1) j} \mathrm{e}^{\mathrm{i}(n-m) \alpha_{j k}} H_{n-m}\left(k_{0} R_{j k}\right)=-I_{k}^{(1)} \mathrm{e}^{\mathrm{i} m(\pi / 2-\beta)},
$$

where $m=-\infty, \ldots, \infty, k=1, \ldots, N_{c}$ and $I_{k}^{(1)}=\mathrm{e}^{\mathrm{i} k_{0}\left[X_{k} \cos \beta+Y_{k} \sin \beta\right]}$ is the phase correction accounting for the position $\left(X_{k}, Y_{k}\right)$ of each cylinder.

In the vicinity of the cylinders, Graf's theorem together with equation (3.5) can be used to write the total potential $\varphi^{(1)}=\varphi_{D}^{(1)}+\varphi_{I}^{(1)}$ in terms of the local coordinates $\left(r_{k}, \theta_{k}, z\right)$. The final simple expression is

$$
\varphi^{(1)}\left(r_{k}, \theta_{k}, z\right)=f_{0}^{(1)}(z) \sum_{m=-\infty}^{\infty} A_{m}^{k}\left[Z_{m 0}^{(1) k} H_{m}\left(k_{0} r_{k}\right)-J_{m}\left(k_{0} r_{k}\right)\right] \mathrm{e}^{\mathrm{i} m \theta_{k}}, \quad r_{k}<R_{j k} \forall j \neq k .
$$

\subsection{Second-order diffraction potential}

The second-order diffraction potential $\varphi_{D}^{(2)}$ is now further decomposed into two parts: $\varphi_{D}^{(2)}=\varphi_{D I}^{(2)}+\varphi_{D D}^{(2)}$. The potential $\varphi_{D I}^{(2)}$ is chosen to satisfy the homogeneous condition on the free surface and a non-homogeneous condition on the cylinders; while the potential $\varphi_{D D}^{(2)}$ satisfies a non-homogeneous condition on the free surface and a homogeneous one on the cylinders.

\subsubsection{Potential $\varphi_{D I}^{(2)}$}

Due to the specified homogeneous condition on the free surface, this potential satisfies a similar BVP to that satisfied by the first-order potential, $\varphi_{D}^{(1)}$, so that the same method can be used. The only difference is that the solution for the potential $\varphi_{D I}^{(2)}$, in addition to the propagating wave part associated with the Hankel functions $H_{m}$, will also include evanescent (local) wave components associated with modified Bessel functions $K_{m}$ of the second kind. 


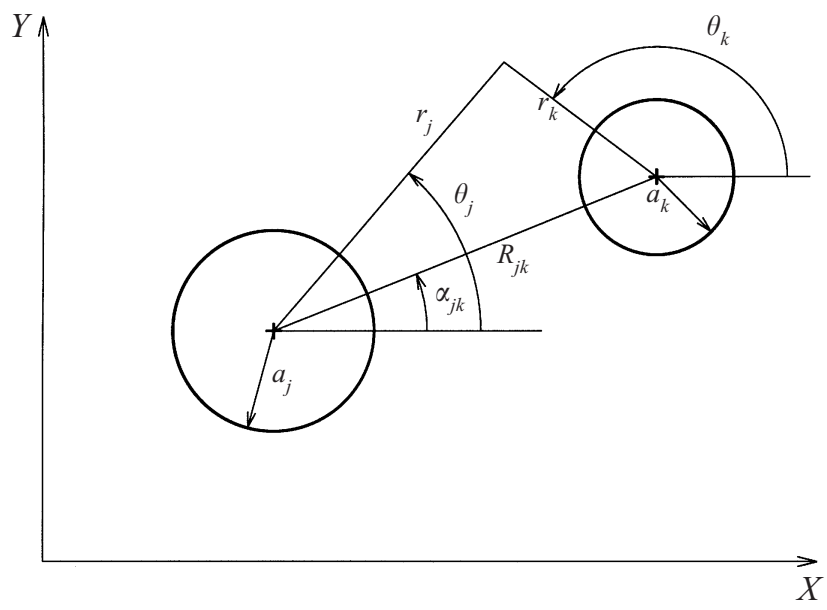

FigURE 1. Basic configuration.

We assume the following eigenfunction expansion for $\varphi_{D I}^{(2)}$ :

$$
\varphi_{D I}^{(2)}=\sum_{k=1}^{N_{c}} \sum_{m=-\infty}^{\infty}\left[f_{0}^{(2)}(z) D_{m 0}^{k} Z_{m 0}^{(2) k} H_{m}\left(\kappa_{0} r_{k}\right)+\sum_{l=1}^{\infty} f_{l}^{(2)}(z) D_{m l}^{k} Z_{m l}^{(2) k} K_{m}\left(\kappa_{l} r_{k}\right)\right] \mathrm{e}^{\mathrm{i} m \theta_{k}},
$$

where $\kappa_{i}(i=0,1 \ldots)$ are solutions of $4 v=\kappa_{0} \tanh \kappa_{0} h=-\kappa_{l} \tan \kappa_{l} h$, and

$$
\left.\begin{array}{rl}
Z_{m 0}^{(2) k} & =\frac{J_{m}^{\prime}\left(\kappa_{0} a_{k}\right)}{H_{m}^{\prime}\left(\kappa_{0} a_{k}\right)}, \quad Z_{m l}^{(2) k}=\frac{I_{m}^{\prime}\left(\kappa_{l} a_{k}\right)}{K_{m}^{\prime}\left(\kappa_{l} a_{k}\right)}, \\
f_{0}^{(2)}(z) & =\frac{\cosh \kappa_{0}(z+h)}{\cosh \kappa_{0} h}, \quad f_{l}^{(2)}(z)=\frac{\cos \kappa_{l}(z+h)}{\cos \kappa_{l} h} .
\end{array}\right\}
$$

$I_{m}$ is the modified Bessel function of the first kind. After applying the boundary condition on each cylinder $\left(\partial \varphi_{D I}^{(2)} / \partial n=-\partial \varphi_{I}^{(2)} / \partial n\right)$, we obtain the following system of equations for the unknown coefficients $D_{m i}^{k}$ :

$$
\begin{gathered}
D_{m 0}^{k}+\sum_{j \neq k}^{N_{c}} \sum_{n=-\infty}^{\infty} D_{n 0}^{j} Z_{n 0}^{(2) j} \mathrm{e}^{\mathrm{i}(n-m) \alpha_{j k}} H_{n-m}\left(\kappa_{0} R_{j k}\right)=\gamma_{m 0}^{k}, \\
D_{m l}^{k}+(-1)^{m} \sum_{j \neq k}^{N_{c}} \sum_{n=-\infty}^{\infty} D_{n l}^{j} Z_{n l}^{(2) j} \mathrm{e}^{\mathrm{i}(n-m) \alpha_{j k}} K_{n-m}\left(\kappa_{l} R_{j k}\right)=\gamma_{m l}^{k},
\end{gathered}
$$

where $m=-\infty, \ldots, \infty, k=1, \ldots, N_{c}, l=1, \ldots, \infty$; and

$$
\begin{aligned}
\gamma_{m 0}^{k} & =-\frac{3 \mathrm{i} \omega v A^{2}}{2 \sinh ^{2} k_{0} h} I_{k}^{(2)} \frac{4 k_{0} C_{0}^{(2)}}{\kappa_{0}\left(4 k_{0}^{2}-\kappa_{0}^{2}\right)} \mathrm{e}^{\mathrm{i} m(\pi / 2-\beta)} \frac{J_{m}^{\prime}\left(2 k_{0} a_{k}\right)}{J_{m}^{\prime}\left(\kappa_{0} a_{k}\right)}, \\
\gamma_{m l}^{k} & =-\frac{3 \mathrm{i} \omega v A^{2}}{2 \sinh ^{2} k_{0} h} I_{k}^{(2)} \frac{4 k_{0} C_{l}^{(2)}}{\kappa_{l}\left(4 k_{0}^{2}+\kappa_{l}^{2}\right)} \mathrm{e}^{\mathrm{i} m(\pi / 2-\beta)} \frac{J_{m}^{\prime}\left(2 k_{0} a_{k}\right)}{I_{m}^{\prime}\left(\kappa_{l} a_{k}\right)},
\end{aligned}
$$

with $I_{k}^{(2)}=\mathrm{e}^{2 \mathrm{i} k_{0}\left(X_{k} \cos \beta+Y_{k} \sin \beta\right)}$ and $C_{i}^{(2)}=\left[2 \int_{-h}^{0} f_{i}^{(2)}(z) f_{i}^{(2)}(z) \mathrm{d} z\right]^{-1}$. 
As in the case of the first-order potential in equation (3.6), this expression can be simplified near the cylinders, $r_{k}<R_{j k}, \forall j \neq k$ :

$$
\begin{aligned}
\varphi_{D I}^{(2)}\left(r_{k}, \theta_{k}, z\right)= & \sum_{m=-\infty}^{\infty}\left\{f_{0}^{(2)}(z)\left[D_{m 0}^{k} Z_{m 0}^{(2) k} H_{m}\left(\kappa_{0} r_{k}\right)+\left(\gamma_{m 0}^{k}-D_{m 0}^{k}\right) J_{m}\left(\kappa_{0} r_{k}\right)\right]\right. \\
& \left.+\sum_{l=1}^{\infty} f_{l}^{(2)}(z)\left[D_{m l}^{k} Z_{m l}^{(2) k} K_{m}\left(\kappa_{l} r_{k}\right)+\left(\gamma_{m l}^{k}-D_{m l}^{k}\right) I_{m}\left(\kappa_{l} r_{k}\right)\right]\right\} \mathrm{e}^{\mathrm{i} m \theta_{k}} .
\end{aligned}
$$

\subsubsection{Potential $\varphi_{D D}^{(2)}$}

This part of the potential is the most difficult to evaluate because of the nonhomogeneous condition on the free surface. We start by writing Green's theorem for a point outside the fluid domain. By using a classical wave source Green function at frequency $2 \omega$, satisfying the homogeneous free surface boundary condition obtained from (2.5), we can write

$$
\iint_{S_{B}} \varphi_{D D}^{(2)} \frac{\partial G}{\partial n} \mathrm{~d} S=\iint_{S_{F}} G Q_{D}^{(2)} \mathrm{d} S
$$

there being no contribution from the integral at infinity as shown by Malenica \& Molin (1995). $Q_{D}^{(2)}$ is the right-hand-side term in the free surface condition (2.5) from which the contributions due to the direct products of the incident wave terms have been removed. In the case of bottom-mounted circular cylinders it can be expressed as

$$
Q_{D}^{(2)}=\frac{\mathrm{i} \omega}{2 g}\left(3 v^{2}-k_{0}^{2}\right)\left(\varphi_{D}^{(1)} \varphi_{D}^{(1)}+2 \varphi_{I}^{(1)} \varphi_{D}^{(1)}\right)+\frac{\mathrm{i} \omega}{g}\left(\nabla_{0} \varphi_{D}^{(1)} \nabla_{0} \varphi_{D}^{(1)}+2 \nabla_{0} \varphi_{I}^{(1)} \nabla_{0} \varphi_{D}^{(1)}\right)
$$

where $\nabla_{0}$ is the horizontal gradient operator $(\partial / \partial x, \partial / \partial y)$. The Green function $G\left(r_{k}, \theta_{k}, z ; \rho_{k}, \vartheta_{k}, \zeta\right)$ is written (cf. Fenton 1978) in the eigenfunction expansion form:

$$
\begin{aligned}
G= & \sum_{m=-\infty}^{\infty}\left\{-\frac{\mathrm{i}}{2} C_{0}^{(2)}\left(\begin{array}{c}
H_{m}\left(\kappa_{0} r_{k}\right) J_{m}\left(\kappa_{0} \rho_{k}\right) \\
J_{m}\left(\kappa_{0} r_{k}\right) H_{m}\left(\kappa_{0} \rho_{k}\right)
\end{array}\right) f_{0}^{(2)}(z) f_{0}^{(2)}(\zeta)\right. \\
& \left.-\frac{1}{\pi} \sum_{n=1}^{\infty} C_{n}^{(2)}\left(\begin{array}{l}
K_{m}\left(\kappa_{n} r_{k}\right) I_{m}\left(\kappa_{n} \rho_{k}\right) \\
I_{m}\left(\kappa_{n} r_{k}\right) K_{m}\left(\kappa_{n} \rho_{k}\right)
\end{array}\right) f_{n}^{(2)}(z) f_{n}^{(2)}(\zeta)\right\} \mathrm{e}^{\mathrm{i} m\left(\theta_{k}-\theta_{k}\right)}, \quad\left(\begin{array}{l}
r_{k}>\rho_{k} \\
r_{k}<\rho_{k}
\end{array}\right) .
\end{aligned}
$$

The potential $\varphi_{D D}^{(2)}$ on the $k$ th cylinder is also assumed in the eigenfunction expansion form

$$
\varphi_{D D}^{(2) k}\left(a_{k}, \theta_{k}, z\right)=\sum_{m=-\infty}^{\infty}\left[B_{m 0}^{k} f_{0}^{(2)}(z)+\sum_{l=1}^{\infty} B_{m l}^{k} f_{l}^{(2)}(z)\right] \mathrm{e}^{\mathrm{i} i \theta_{k}} .
$$

We substitute these into equation (3.14) for a point inside the cylinder $k$, i.e. for $r_{k}=a_{k}-\delta, 0<\delta \leqslant a_{k}$, and carry out the integration with respect to $\zeta$, using the orthogonality of the functions $f_{n}^{(2)}(\zeta)$. Using Graf's addition theorem for the Bessel functions, exploiting the orthogonality of the functions $\mathrm{e}^{\mathrm{i} m \theta}$, and rearranging the different terms, we obtain the following system of equations for the unknown 
coefficients $B_{m i}^{k}$ :

$$
\begin{array}{r}
B_{m 0}^{k}+\sum_{j \neq k}^{N_{c}} \frac{a_{j}}{a_{k}} \sum_{n=-\infty}^{\infty} B_{n 0}^{j} \frac{J_{n}^{\prime}\left(\kappa_{0} a_{j}\right)}{H_{m}^{\prime}\left(\kappa_{0} a_{j}\right)} H_{n-m}\left(\kappa_{0} R_{j k}\right) \mathrm{e}^{\mathrm{i}(n-m) \alpha_{j k}} \\
=-\frac{C_{0}^{(2)}}{\pi a_{k} \kappa_{0} H_{m}^{\prime}\left(\kappa_{0} a_{k}\right)} \iint_{S_{F}} H_{m}\left(\kappa_{0} \rho_{k}\right) \mathrm{e}^{-\mathrm{i} m \vartheta_{k}} Q_{D}^{(2)}\left(\rho_{k}, \vartheta_{k}\right) \mathrm{d} S, \\
B_{m l}^{k}+(-1)^{m} \sum_{j \neq k}^{N_{c}} \frac{a_{j}}{a_{k}} \sum_{n=-\infty}^{\infty} B_{n l}^{j} \frac{I_{n}^{\prime}\left(\kappa_{l} a_{j}\right)}{K_{m}^{\prime}\left(\kappa_{l} a_{j}\right)} K_{n-m}\left(\kappa_{l} R_{j k}\right) \mathrm{e}^{\mathrm{i}(n-m) \alpha_{j k}} \\
=-\frac{C_{l}^{(2)}}{\pi a_{k} \kappa_{l} K_{m}^{\prime}\left(\kappa_{l} a_{k}\right)} \iint_{S_{F}} K_{m}\left(\kappa_{l} \rho_{k}\right) \mathrm{e}^{-\mathrm{i} m \vartheta_{k}} Q_{D}^{(2)}\left(\rho_{k}, \vartheta_{k}\right) \mathrm{d} S,
\end{array}
$$

where $m=-\infty, \ldots, \infty, k=1, \ldots, N_{c}, l=1, \ldots, \infty$.

This completes the solution for the potential $\varphi_{D D}^{(2)}$ on the cylinders. As we can see, the solution is written in the form of eigenfunction expansions, with the eigenfunctions in the $z$-direction satisfying the homogeneous free surface condition, even though the required condition on the free surface is non-homogeneous. This important point was discussed by Chau \& Eatock Taylor (1992) and Eatock Taylor \& Huang (1997a), who showed that the non-homogeneous condition on the free surface is nevertheless satisfied in the limiting sense as $z \rightarrow 0^{-}$. The consequence of this representation is slow convergence of the infinite summation over $l$ in equation (3.17) and the appearance of a logarithmic singularity. Furthermore, special care is required when calculating the potential on the free surface.

Once the potential on the cylinders has been found, the solution at any point in the fluid can be formulated using Green's theorem. The resulting expression is

$$
\begin{aligned}
\varphi_{D D}^{(2)}= & \sum_{j=1}^{N_{c}} 2 \pi a_{j} \sum_{m=-\infty}^{\infty}\left[B_{m 0}^{j} \frac{1}{4} \mathrm{i} \kappa_{0} J_{m}^{\prime}\left(\kappa_{0} a_{j}\right) f_{0}^{(2)}(z) H_{m}\left(\kappa_{0} r_{j}\right)\right. \\
& \left.+\sum_{n=1}^{\infty} B_{m n}^{j} \frac{\kappa_{n}}{2 \pi} I_{m}^{\prime}\left(\kappa_{n} a_{j}\right) f_{n}^{(2)}(z) K_{m}\left(\kappa_{n} r_{j}\right)\right] \mathrm{e}^{\mathrm{i} m \theta_{j}}-\iint_{S_{F}} G Q_{D}^{(2)} \mathrm{d} S .
\end{aligned}
$$

As above (cf. equations (3.6) and (3.13)), the use of Graf's theorem together with equations (3.18) and (3.19) leads to simplified expressions for the potential near the cylinders $r_{k}<R_{j k}, \forall j \neq k$ :

$$
\begin{aligned}
\varphi_{D D}^{(2)}= & \sum_{m=-\infty}^{\infty}\left\{\frac{\mathrm{i} \pi a_{k} \kappa_{0}}{2} f_{0}^{(2)}(z) B_{m 0}^{k}\left[J_{m}^{\prime}\left(\kappa_{0} a_{k}\right) H_{m}\left(\kappa_{0} r_{k}\right)-H_{m}^{\prime}\left(\kappa_{0} a_{k}\right) J_{m}\left(\kappa_{0} r_{k}\right)\right]\right. \\
& +\sum_{l=1}^{\infty} a_{k} \kappa_{l} f_{l}^{(2)}(z) B_{m l}^{k}\left[I_{m}^{\prime}\left(\kappa_{l} a_{k}\right) K_{m}\left(\kappa_{l} r_{k}\right)-K_{m}^{\prime}\left(\kappa_{l} a_{k}\right) I_{m}\left(\kappa_{l} r_{k}\right)\right] \\
& +\int_{0}^{r_{k}}\left\{\mathrm{i} \pi C_{0}^{(2)} f_{0}^{(2)}(z)\left[H_{m}\left(\kappa_{0} r_{k}\right) J_{m}\left(\kappa_{0} \rho_{k}\right)-J_{m}\left(\kappa_{0} r_{k}\right) H_{m}\left(\kappa_{0} \rho_{k}\right)\right]\right. \\
& \left.+2 \sum_{l=1}^{\infty} C_{l}^{(2)} f_{l}^{(2)}(z)\left[K_{m}\left(\kappa_{l} r_{k}\right) I_{m}\left(\kappa_{l} \rho_{k}\right)-I_{m}\left(\kappa_{l} r_{k}\right) K_{m}\left(\kappa_{l} \rho_{k}\right)\right]\right\} \\
& \left.\times Q_{D m}^{(2)}\left(\rho_{k}\right) \rho_{k} \mathrm{~d} \rho_{k}\right\} \mathrm{e}^{\mathrm{i} m \theta_{k}} .
\end{aligned}
$$


An important feature of equation (3.21) is that once the coefficients $B_{m i}^{k}$ have been obtained (by solving equations (3.18) and (3.19)), the potential at any arbitrary point in the vicinity of the cylinders can be obtained without the need for the infinite quadrature indicated in equation (3.20). We may also note that in the limiting case of a single cylinder the expressions (3.18) (3.19), and (3.21) reduce to the expressions given by Chau \& Eatock Taylor (1992), or Malenica \& Molin (1995).

\section{Numerical implementation}

4.1. Interaction effects and the free surface integrals

Calculation of the first-order diffraction potential $\varphi_{D}^{(1)}$, and the first part of the second-order diffraction potential $\varphi_{D I}^{(2)}$, needs no special treatment, and the numerical implementation is straightforward. In the case of $\varphi_{D I}^{(2)}$, however, much computational time can be saved if the interaction effects are neglected after some value of $l$, i.e. for $\kappa_{l}$ sufficiently large. This is justified by the fact that after some value of $l$ the interaction effects become negligibly small, which can be deduced from equation (3.10). In fact, for large values of $\kappa_{l}$, the product $Z_{n l}^{(2) j} K_{n-m}\left(\kappa_{l} R_{j k}\right)$ behaves asymptotically as

$$
\lim _{l \rightarrow \infty} \frac{I_{n}^{\prime}\left(\kappa_{l} a_{j}\right)}{K_{n}^{\prime}\left(\kappa_{l} a_{j}\right)} K_{n-m}\left(\kappa_{l} R_{j k}\right)=-\frac{\mathrm{e}^{\kappa_{l}\left(2 a_{j}-R_{j k}\right)}}{\sqrt{2 \pi \kappa_{l} R_{j k}}} .
$$

Knowing that $\kappa_{l}$ is a monotonically increasing function of $l$, with $\kappa_{l} \rightarrow l \pi / h$ for $l \rightarrow \infty$, we can adopt a simple rule for choosing $L=l_{\max }$ We specify

$$
\kappa_{L}>\frac{-\ln \epsilon_{1}}{R_{j k}-2 a_{j}},
$$

where in our double precision calculations the coefficient $\epsilon_{1}$ is choosen to be $10^{-12}$. Neglect of the interaction effects implies that the coefficients $D_{m l}^{k}$ become equal to $\gamma_{m l}^{k}$ for $l>L$.

In the evaluation of the potential $\varphi_{D D}^{(2)}$, several simplifications are also possible. The main difficulty in solving the system of equations (3.18) and (3.19) is associated with the evaluation of the free surface integrals, which must exclude the cylinder water planes. In order to minimize the effort in performing the two-dimensional integration, the following procedure can be adopted. We refer to the notation defined in figure 2 . In the inner $\left(r_{0} \leqslant R_{b}\right)$ and outer $\left(r_{0} \geqslant R_{e}\right)$ regions we can use Graf's addition theorem and write the first-order diffraction potential $\varphi_{D}^{(1)}$ in terms of the global coordinates $\left(r_{0}, \theta_{0}\right)$ :

$$
\begin{aligned}
& \left.\varphi_{D}^{(1)}\right|_{r_{0} \leqslant R_{b}}=f_{0}^{(1)}(z) \sum_{m=-\infty}^{\infty}\left\{\sum_{k=1}^{N_{c}} \sum_{n=-\infty}^{\infty} A_{n}^{k} Z_{n 0}^{(1) k} H_{m-n}\left(k_{0} R_{0 k}\right) \mathrm{e}^{\mathrm{i}(n-m) \alpha_{0 k}}\right\} J_{m}\left(k_{0} r_{0}\right) \mathrm{e}^{\mathrm{i} m \theta_{0}}, \\
& \left.\varphi_{D}^{(1)}\right|_{r_{0} \geqslant R_{e}}=f_{0}^{(1)}(z) \sum_{m=-\infty}^{\infty}\left\{\sum_{k=1}^{N_{c}} \sum_{n=-\infty}^{\infty} A_{n}^{k} Z_{n 0}^{(1) k} J_{m-n}\left(k_{0} R_{0 k}\right) \mathrm{e}^{\mathrm{i}(n-m) \alpha_{0 k}}\right\} H_{m}\left(k_{0} r_{0}\right) \mathrm{e}^{\mathrm{i} m \theta_{0}} .
\end{aligned}
$$

The forcing function $Q_{D}^{(2)}$ can then be easily written in the form of a Fourier series expansion:

$$
Q_{D}^{(2)}=\sum_{m=-\infty}^{\infty} Q_{D m}^{(2)}\left(r_{0}\right) \mathrm{e}^{\mathrm{i} m \theta_{0}} .
$$




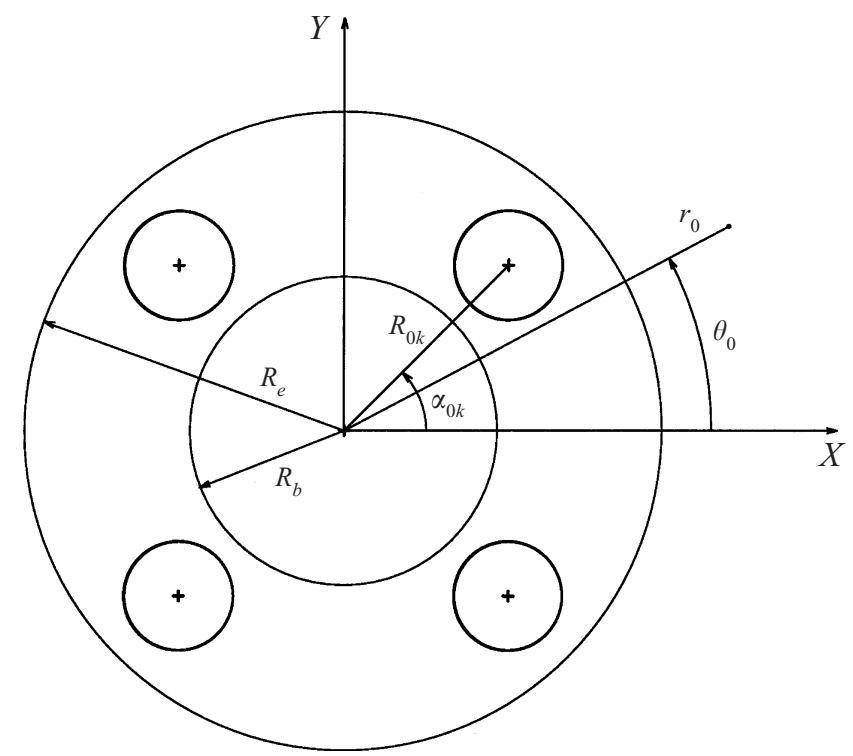

FIGURE 2. Different regions for integration on the free surface.

At the same time we use Graf's theorem to develop the term $H_{m}\left(\kappa_{0} \rho_{k}\right) \mathrm{e}^{-\mathrm{i} m \vartheta_{k}}$ as follows:

$$
\begin{aligned}
H_{m}\left(\kappa_{0} \rho_{k}\right) \mathrm{e}^{-\mathrm{i} m \vartheta_{k}} & =\sum_{n=-\infty}^{\infty} H_{n-m}\left(\kappa_{0} R_{0 k}\right) \mathrm{e}^{\mathrm{i}(n-m) \alpha_{0 k}} J_{n}\left(\kappa_{0} \rho_{0}\right) \mathrm{e}^{-\mathrm{i} n \vartheta_{0}} \\
& =\sum_{n=-\infty}^{\infty} \alpha_{m n}^{k 0} J_{n}\left(\kappa_{0} \rho_{0}\right) \mathrm{e}^{-\mathrm{i} n \vartheta_{0}}, \quad\left(\rho_{0} \leqslant R_{0 k}\right), \\
H_{m}\left(\kappa_{0} \rho_{k}\right) \mathrm{e}^{-\mathrm{i} m \vartheta_{k}} & =\sum_{n=-\infty}^{\infty} J_{n-m}\left(\kappa_{0} R_{0 k}\right) \mathrm{e}^{\mathrm{i}(n-m) \alpha_{0 k}} H_{n}\left(\kappa_{0} \rho_{0}\right) \mathrm{e}^{-\mathrm{i} n \vartheta_{0}} \\
& =\sum_{n=-\infty}^{\infty} \beta_{m n}^{k 0} H_{n}\left(\kappa_{0} \rho_{0}\right) \mathrm{e}^{-\mathrm{i} n \vartheta_{0}}, \quad\left(\rho_{0} \geqslant R_{0 k}\right) .
\end{aligned}
$$

The free surface integral is now divided into three parts:

$$
\iint_{S_{F}}=\int_{0}^{2 \pi} \int_{0}^{R_{b}}+\iint_{S_{d}}+\int_{0}^{2 \pi} \int_{R_{e}}^{\infty}
$$

where $S_{d}$ is the surface between $R_{b}$ and $R_{e}$ without cylinder surfaces. In the first and third integral, the integration with respect to $\vartheta_{0}$ can be carried out explicitly. For the propagating mode

$$
\begin{aligned}
& \int_{0}^{2 \pi} \int_{0}^{R_{b}} H_{m}\left(\kappa_{0} \rho_{k}\right) \mathrm{e}^{-\mathrm{i} m \vartheta_{k}} Q_{D}^{(2)} \rho_{0} \mathrm{~d} \rho_{0} \mathrm{~d} \vartheta_{0}=2 \pi \sum_{n=-\infty}^{\infty} \alpha_{m n}^{k 0} \int_{0}^{R_{b}} J_{n}\left(\kappa_{0} \rho_{0}\right) Q_{D n}^{(2)}\left(\rho_{0}\right) \rho_{0} \mathrm{~d} \rho_{0} \\
& \int_{0}^{2 \pi} \int_{R_{e}}^{\infty} H_{m}\left(\kappa_{0} \rho_{k}\right) \mathrm{e}^{-\mathrm{i} m \vartheta_{k}} Q_{D}^{(2)} \rho_{0} \mathrm{~d} \rho_{0} \mathrm{~d} \vartheta_{0},=2 \pi \sum_{n=-\infty}^{\infty} \beta_{m n}^{k 0} \int_{R_{e}}^{\infty} H_{n}\left(\kappa_{0} \rho_{0}\right) Q_{D n}^{(2)}\left(\rho_{0}\right) \rho_{0} \mathrm{~d} \rho_{0}
\end{aligned}
$$

and a similar procedure applies for evanescent parts of the solution. In this way 
we reduce these two-dimensional surface integrals to one-dimensional line integrals and, at the same time, the most difficult integral (from $R_{e}$ to $\infty$ ) is put in the same form as in the single cylinder case so that the same method can be used for its evaluation. Furthermore, the integrations in both inner and outer regions become the same for all cylinders, which is very important from a numerical point of view. The only full two-dimensional integration needed is that over the surface $S_{d}$, and since this surface is relatively small, it can be done with relatively small computational effort. For the results below, the numerical integration step length in both the radial and circumferential directions was based on the relation

$$
L_{T}=\frac{\pi}{N_{T}\left(2 k_{0}+\kappa_{0}\right)} .
$$

Numerical tests indicated that the value of $N_{T}$ should be chosen in the range 15-25.

As discussed above in connection with $\varphi_{D I}^{(2)}$, we may also neglect the influence of interaction effects on $\varphi_{D D}^{(2)}$ for sufficiently large values of $l$. The neglect of the effects of interaction for $l>L$ then implies, from equation (3.19), that the coefficients $B_{m l}^{k}$ become

$$
B_{m l}^{k}=-\frac{C_{l}^{(2)}}{\pi a_{k} \kappa_{l} K_{m}^{\prime}\left(\kappa_{l} a_{k}\right)} \int_{0}^{2 \pi} \int_{a_{k}}^{R_{k}} K_{m}\left(\kappa_{l} \rho_{k}\right) Q_{D m}^{(2)}\left(\rho_{k}\right) \mathrm{e}^{-\mathrm{i} m \vartheta_{k}} \mathrm{~d} S, \quad l>L,
$$

where $a_{k}<R_{k}<R_{j k}, \forall j \neq k$. Since the first-order potential in the vicinity of the $k$ th cylinder can be written in the form of equation (3.6), and $R_{k}$ can be chosen sufficiently close to the body, the calculation of the coefficients $B_{m l}^{k}$ becomes very similar to the single cylinder case (Eatock Taylor \& Huang 1997b). Hence much computational time can be saved. In view of the efficient evaluation of the forcing term $Q_{D m}^{(2)}$, using equation (3.6) for the first-order potential in equation (3.15), it is found that the following condition must be satisfied for the chosen value of $L$ :

$$
\kappa_{L}>\frac{-\ln \epsilon_{2}}{R_{k}-a_{k}} .
$$

This condition is consistent with requiring the asymptotic value of the modified Bessel function $K_{m}\left(\kappa_{L} \rho_{k}\right)$, for $\rho_{k}=R_{k}$, to be less than $\epsilon_{2}$ times its value for $\rho_{k}=a_{k}$. In our calculations the value of $\epsilon_{2}$ is chosen to be $10^{-5}$ for $R_{k}=R_{j k} / 2$. It should be noted that equation (4.12) is also well suited to evaluation of the second-order potential in the vicinity of the $k$ th cylinder, because the logarithmic singularity can be treated in a similar way as for the single cylinder case.

\subsection{Convergence tests}

In order to demonstrate the convergence of various infinite sums arising in the expressions for the potentials, we chose the example of an array of four cylinders. Each cylinder is centred at the corner of a square with side length equal to six times the radius $a$ of each cylinder. The $z$-axis passes through the centre of the square, and the cylinders are numbered in an anti-clockwise sense, starting with the cylinder placed at the point $(X, Y)=(3 a, 3 a)$. The water depth is $3 a$, and for these convergence tests the non-dimensional wavenumber is $k_{0} a=1.1$ and the wave incidence angle is $\beta=0$. Figure 3 shows $\eta^{(22)}$, which is that part of the second-order free surface elevation due to the second-order potential $\varphi^{(2)}$ over cylinder $2(\theta=\pi$ is the upwave side, and $\theta=3 \pi / 2$ is on the inside of the square for this upwave cylinder). We can see that for this intermediate wave number, eight Fourier harmonics are sufficient to represent the free surface elevation around the cylinder using equations (3.17), 


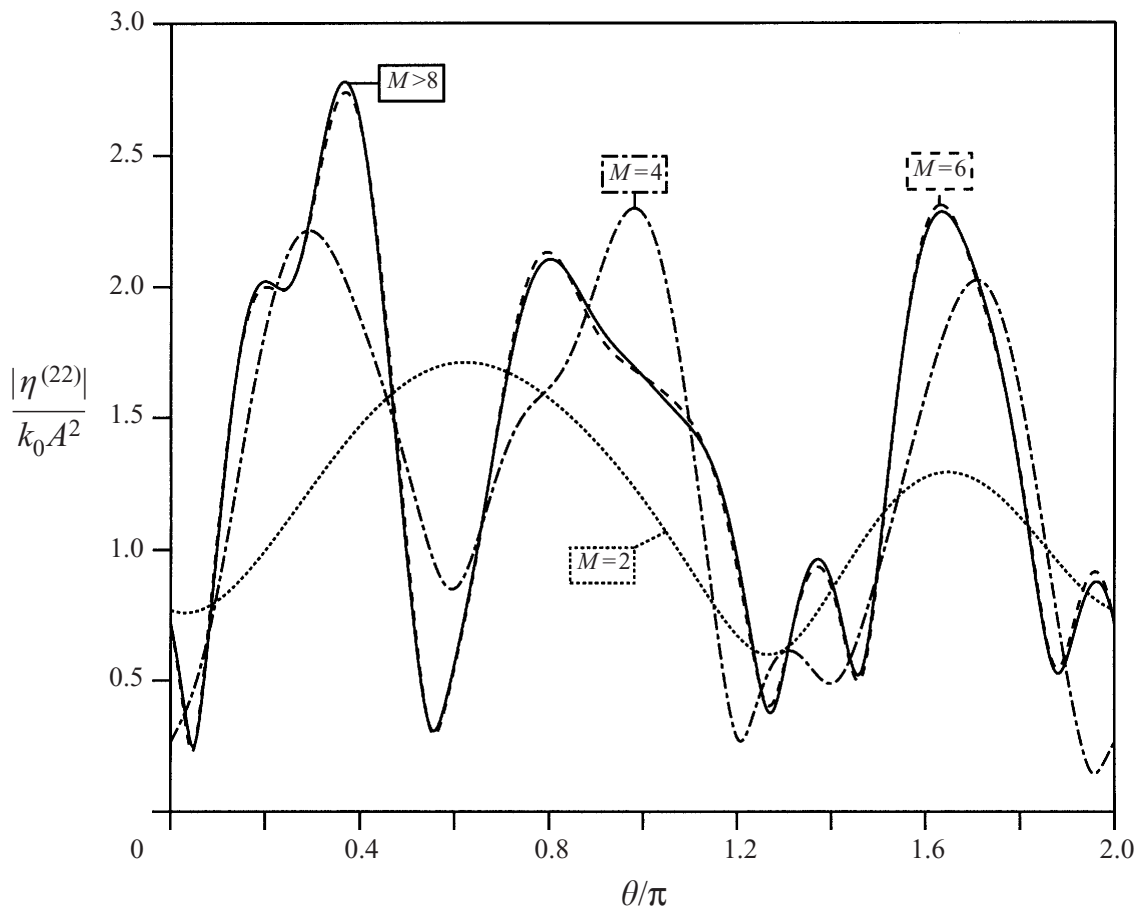

FiguRE 3. Convergence of the Fourier series expansion: second-order free surface run-up, $\left|\eta^{(22)} / k_{0} A^{2}\right|$, around a cylinder in an array, for different numbers of terms $M$.

(3.7) and (3.2). It should be noted however that, in order to calculate the eight terms in the Fourier series expansion (3.17), many more terms should be used in the evaluation of the integrals in expressions (4.10) and (4.10). This is because the use of Graf's theorem in obtaining equations (4.3), (4.4), (4.6) and (4.7) necessitates a sufficient number of terms to provide an accurate transformation of the coordinates. This number depends on the values of $R_{b}$ and $R_{e}$, which are not necessarly equal to $R_{0 k} \pm a_{k}$. For $\left(R_{b}, R_{e}\right)=R_{k} \pm 1.3 a_{k}$, and depending on the wavenumber, we usually need around three times the number of harmonics retained in equation (3.17).

In order to illustrate the convergence of the eigenfunction expansion in the vertical direction, we show in figure 4 the second-order elevation $\eta^{(22)}$ on cylinder 2 at the upwave point $\left(r_{2}, \theta_{2}, z\right)=(a, \pi, 0)$. The calculations are made for $M=10$ Fourier modes, and different numbers $N$ of vertical eigenfunctions. We observe that the convergence is very slow if the logarithmic singularity is not extracted. In this case, up to 500 terms are necessary in order to obtain a converged result, while only 15 terms are needed when the logarithmic singularity is extracted. We should note that this problem of convergence is not present when only the forces are calculated, because the additional integration with respect to $z$ regularizes the summation.

\section{Numerical results and discussion}

Results for the first-order problem have been presented and discussed by Linton \& Evans (1990) and others. In this section we give only the results for the secondorder quantities. We start by presenting some results which validate our approach. In figure 5 we show comparisons for the second-order surge force on a four-column 


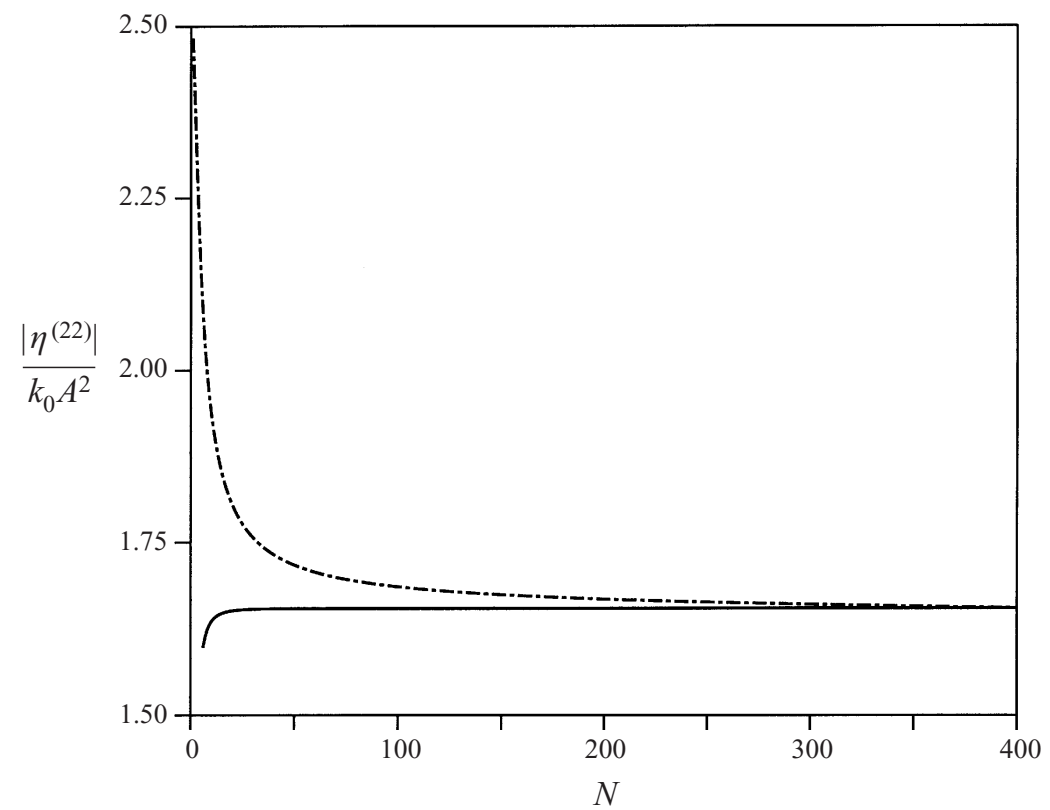

FIGURE 4. Convergence of the eigenfunctions expansion in the $z$-direction: second-order free surface elevation, $\left|\eta^{(22)} / k_{0} A^{2}\right|$, at upwave face of a cylinder in an array, plotted against the number of terms $N$. Full line: results when the logarithmic singularity is extracted; dot-dashed line: results when the summation is carried out directly.
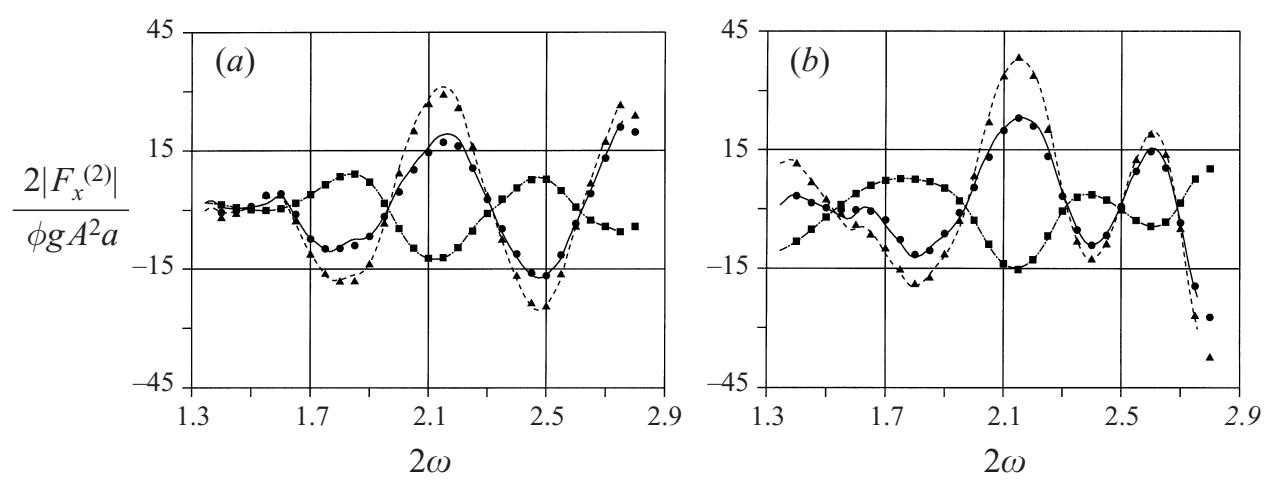

FIGURE 5. (a) Real and $(b)$ imaginary parts of the dimensionless second-order surge force components for an array of four cylinders. Dot-dashed line: $\left|F_{x}^{(21)}\right| / \varrho g A^{2} a$; dashed line: $\left|F_{x}^{(22)}\right| / \varrho g A^{2} a$; full line: $\left|F_{x}^{(2)}\right| / \varrho g A^{2} a$; symbols: results of Chen et al. (1995).

structure in head waves, obtained by the present method, with those obtained by Chen, Molin \& Petitjean (1995) using the indirect approach (with a semi-analytical solution for the 'assisting' radiation potential used in the Haskind relation). The four columns are of $25 \mathrm{~m}$ diameter, placed at the corners of a square of side length $76 \mathrm{~m}$ in water of $130 \mathrm{~m}$ depth. The complete second-order force, $F^{(2)}$, is divided in two parts: the first, $F_{x}^{(12)}$, is associated with the quadratic products of first-order quantities; and the second, $F_{x}^{(22)}$, with the contribution from the second-order potential. The figure shows the variation of these components with frequency. The structure investigated by Chen et al. consisted of cylinders truncated at a draught equal to half the water 

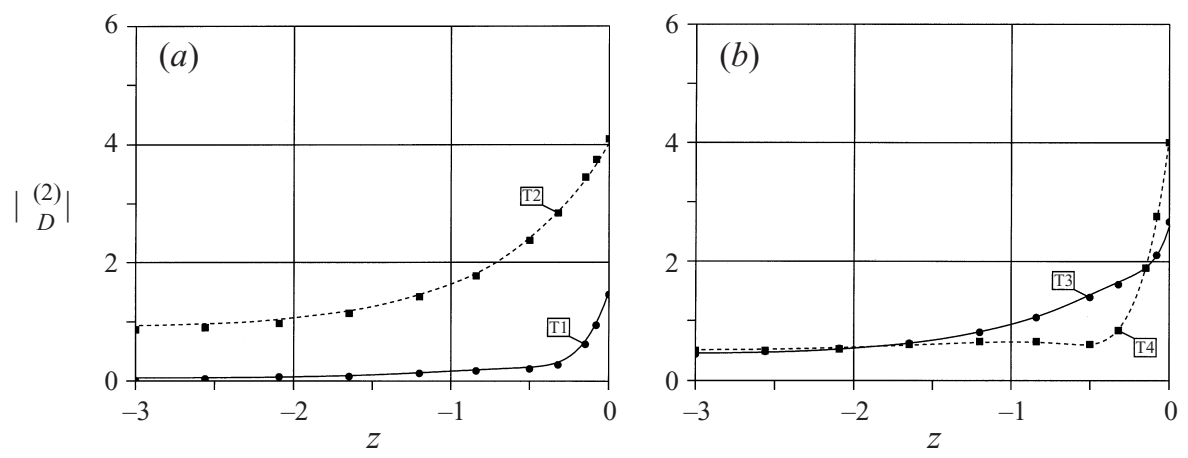

FIGURE 6. Vertical distribution of the second-order diffraction potential down each of two cylinders in tandem, for $k_{0} a=1.4$. Lines: semi-analytical results; symbols: results of Chau (1989).

depth. In both their approach and ours the interaction effects are calculated for complete cylinders extending to the seabed, but the integration of the pressure is performed only over the draught of the cylinders. Thus, as far as the forces are concerned, the only essential difference between the two methods is the treatment of the free surface integral. In Chen et al. (1995) the integration in the vicinity of the cylinders is performed by numerical quadrature, while the integration to infinity is approximated by filtering the oscillations in the integrand. This probably explains the small differences in the results. In figure 5 (where lines correspond to our results and the plotting symbols to those of Chen et al.) two points should be noted. First, due to the different conventions in the two methods, the second-order forces plotted on figure 5 are twice the values which should be obtained from the definition in equation (2.12). Secondly, one can observe small wiggles in these (and some subsequent) curves. These are not thought to be due to lack of convergence.

Comparisons for another case are shown in figure 6. This illustrates the distribution of the second-order potential down the sides of two cylinders in a tandem configuration. These results were first obtained numerically by Chau (1989), using the boundary integral equation technique. Each cylinder has radius $a$, the water depth is $3 a$ and the wave incidence $\beta=0$. The axes of the cylinders are located at $\left(X_{1}, Y_{1}\right)=(2 a, 0)$ and $\left(X_{2}, Y_{2}\right)=(-2 a, 0)$. In figure 6 the vertical distribution of $\left|\varphi_{D}^{(2)}\right|$, for the non-dimensional frequency $k_{0} a=1.4$ is shown for four points: $\mathrm{T} 1=(3 a, 0) ; \mathrm{T} 2=(-3 a, 0) ; \mathrm{T} 3=(a, 0) ;$ and $\mathrm{T} 4=(-a, 0)$. The lines represent the semi-analytical results obtained here, and the symbols correspond to the numerical results from Chau (1989). One can observe the effects of shielding, and the well-known slow depthwise decay of the second-order potential (discussed by Newman 1990 in the context of the deep water case). But of more importance here, we can see very good agreement between the two sets of results. Several other comparisons, not presented here, show a similar level of agreement.

Next we present some results which may be useful in connection with more detailed benchmark studies. Figure 7 shows the circumferential variation of the total secondorder free surface elevation (as defined in equation (2.7)) on two of the cylinders in the four-column configuration considered in figures 3 and 4 . The non-dimensional wavenumber is $k_{0} a=1.1$. Two angles of incidence are investigated, $\beta=0$ and $\beta=\pi / 4$, and the results for an isolated cylinder are also presented for comparison. We can observe that the distribution of elevation on the surface of each cylinder has a completely different shape when the interaction effects are accounted for. A similar 

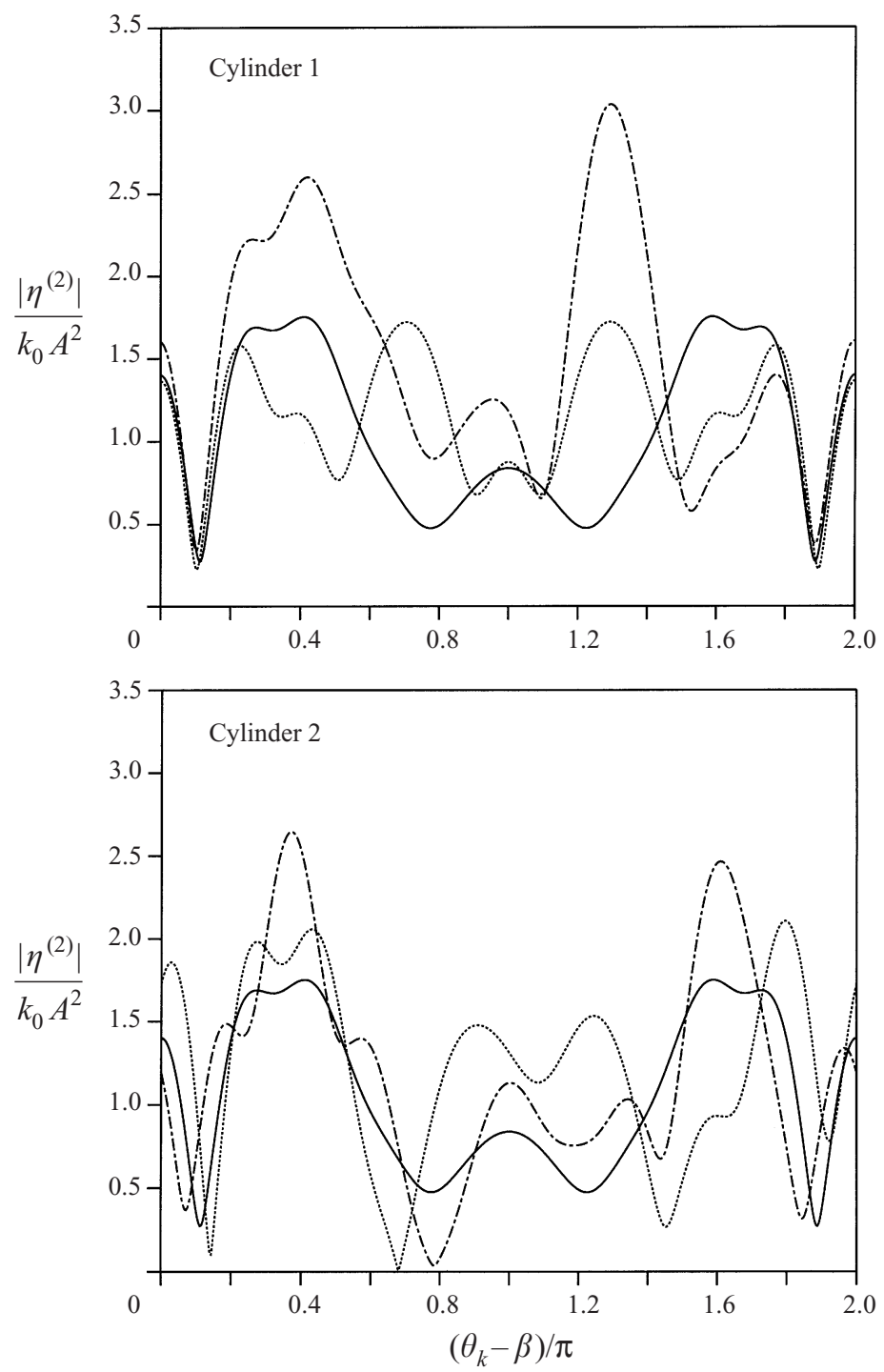

FIGURE 7. Total second-order free surface elevation, $\left|\eta^{(2)}\right| / k_{0} A^{2}$, on the cylinders. Full line: single-cylinder case; dotted line: four-cylinder case for incidence $\beta=0$; dot-dashed line: fourcylinder case for incidence $\beta=\pi / 4$.

conclusion can be drawn from figure 8, where the distributions of the second-order pressure down the downwave sides $(\theta=0)$ of all four cylinders are presented. We again note the slow attenuation of the second-order potential.

The interaction between the cylinders also affects the forces acting on each cylinder. In figure 9 the amplitude of the second-order dimensionless surge force and pitch moment on each cylinder is presented, for the same four-cylinder array as in the previous example. The results for an isolated cylinder are also presented for comparison. It may be observed that throughout almost all of the frequency range plotted, the force and moment on each cylinder of the array is larger than that on the isolated cylinder. 


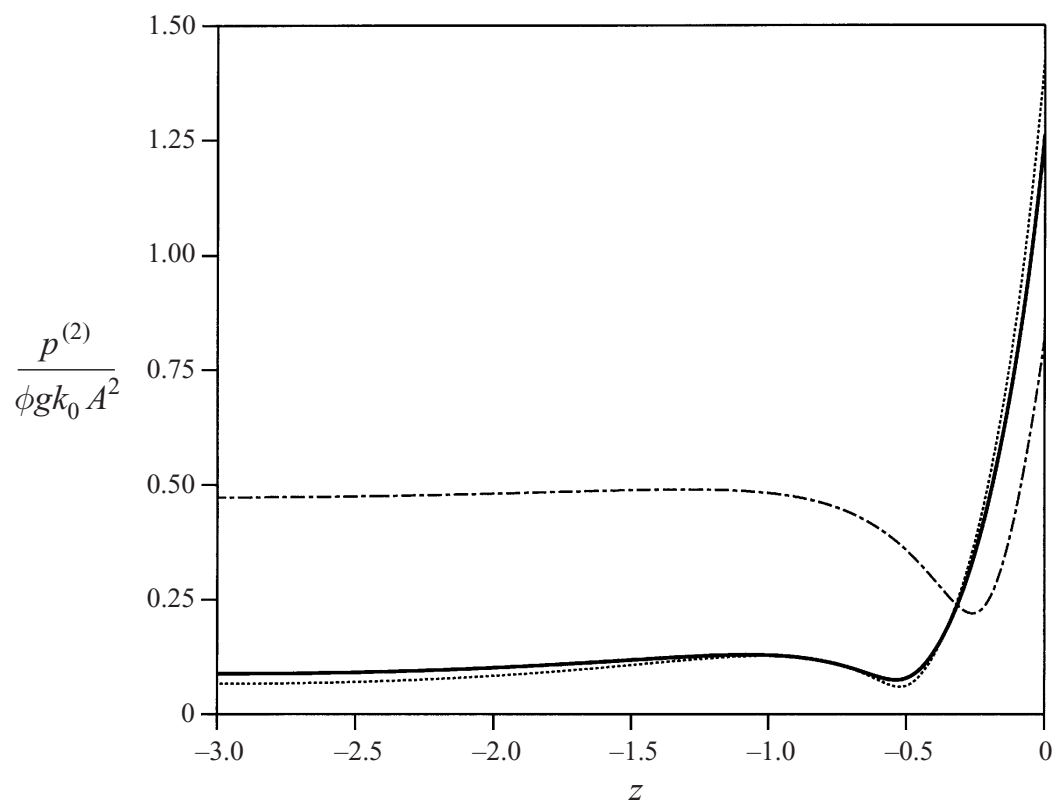

FIGURE 8. Distribution of the second-order pressure, $\left|p^{(2)}\right| / \varrho g k_{0} A^{2}$, down the sides $\left(\theta_{k}=0\right)$ of four cylinders, for a wave incidence $\beta=0$. Full line: results for isolated cylinder; dotted line: first and fourth cylinder; dot-dashed line: second and third cylinder.

In figure 10 some results for different configurations are compared, each in water of depth $3 a$. The four-cylinder configuration is that from the previous example; the three-cylinder configuration is obtained by placing the cylinders at the vertices of an equilateral triangle of side length $6 a$, with the origin at the centre of the triangle and the first cylinder on the positive $x$-axis; the two-cylinder configuration is a tandem configuration with the axes of the cylinders $6 a$ apart on the $x$-axis. The variation with frequency of the average dimensionless second-order force and moment are plotted, i.e. the total force amplitude on the configuration, divided by the number of cylinders. It may be seen that even though the individual forces on the cylinders may be larger for the array than for an individual cylinder (figure 9), the total second-order force may not be, because of phase differences between the cylinders.

Finally, we present results for the free surface elevations in the vicinity of a fourcolumn structure. These highlight the advantages of the analysis described here, as compared with other published analyses of arrays of cylinders which can provide second-order forces but not second-order kinematics. We can anticipate from the findings of Evans \& Porter (1997) that the phenomenon of near-trapping of the firstorder waves would influence the second-order elevations at certain frequencies. Some interesting features of the second-order elevation near an array of cylinders have already been reported by Scolan \& Malenica (1998); they mention near-trapping in this context, but no clear conclusions were reached. We have investigated this further here, obtaining second-order results for two of the configurations examined by Evans $\&$ Porter (1997) in the context of near-trapping at first-order.

We first consider the case where the bottom-mounted cylinders are at the corners of a square of side length $4 a$ (i.e. $a / d=0.5$, where $2 d$ is used by Evans $\&$ Porter to define the side length). The water depth is $3 a$. The origin of the coordinates is 

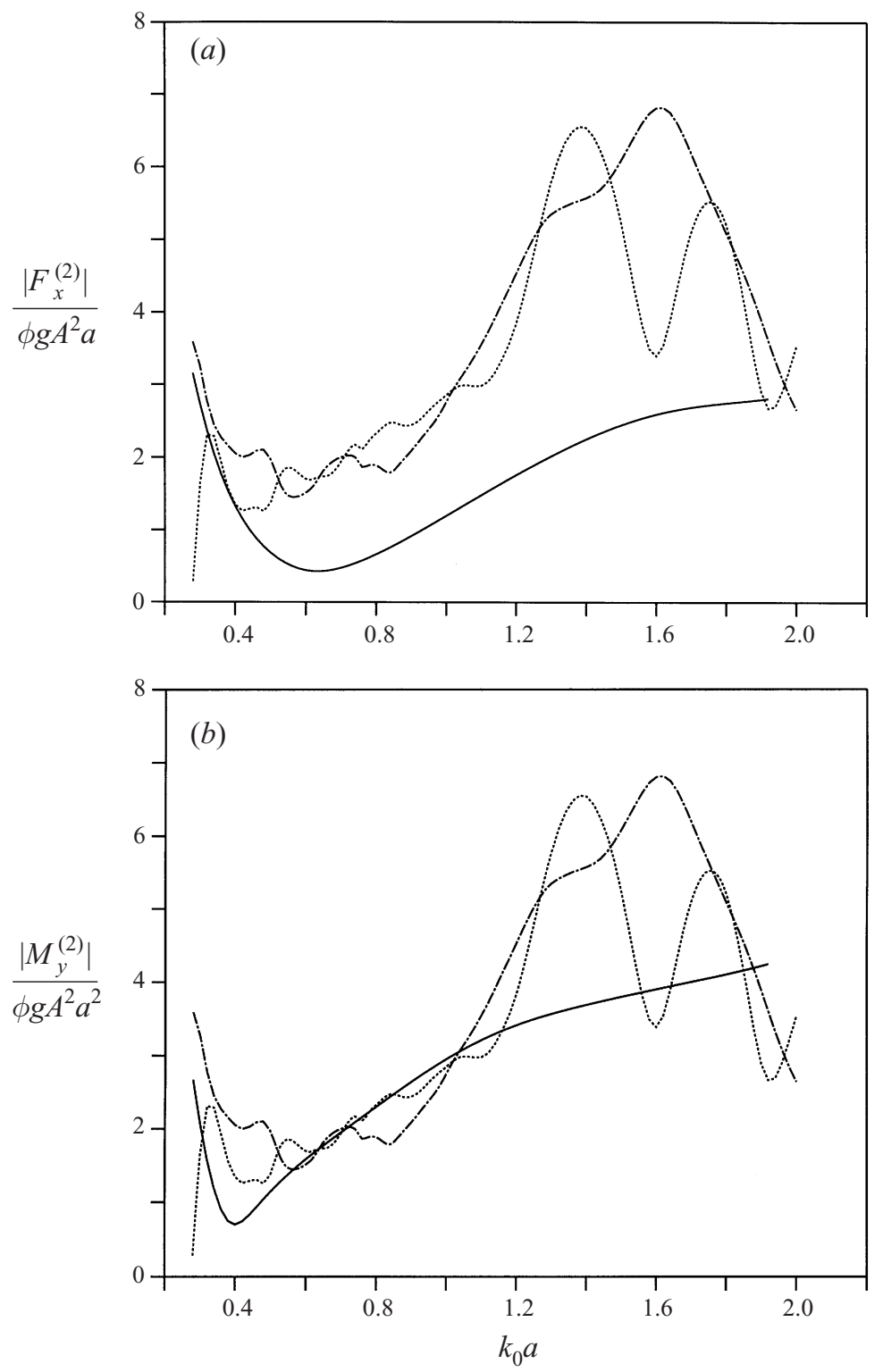

FiguRE 9. Amplitude of $(a)$ the second-order dimensionless surge force and $(b)$ the pitch moment on each cylinder in the four-cylinder configuration for an incidence $\beta=0$. Full line: isolated cylinder; dot-dashed line: first and fourth cylinder; dotted line: second and third cylinder.

taken at the centre of the square, with axes parallel to the sides. Figures 11(a) to $11(d)$ show the components of first- and second-order non-dimensionalised elevation amplitude along the diagonal $x=y$ when the waves are incident along the diagonal $(\beta=\pi / 4)$. The vertical lines identify the positions of the cylinders. Each sub-plot shows results for three wave frequencies: the dotted line is for $k_{0} a=0.754$; the dashed line is for $k_{0} a=0.468$; and the solid line is for $k_{0} a=1.66$. Figure $11(a)$ shows the linear component $\left|\eta^{(1)}\right|$. Figures $11(b)$ and $11(c)$ show $\left|\eta^{(21)}\right|$ and $\left|\eta^{(22)}\right|$, which 

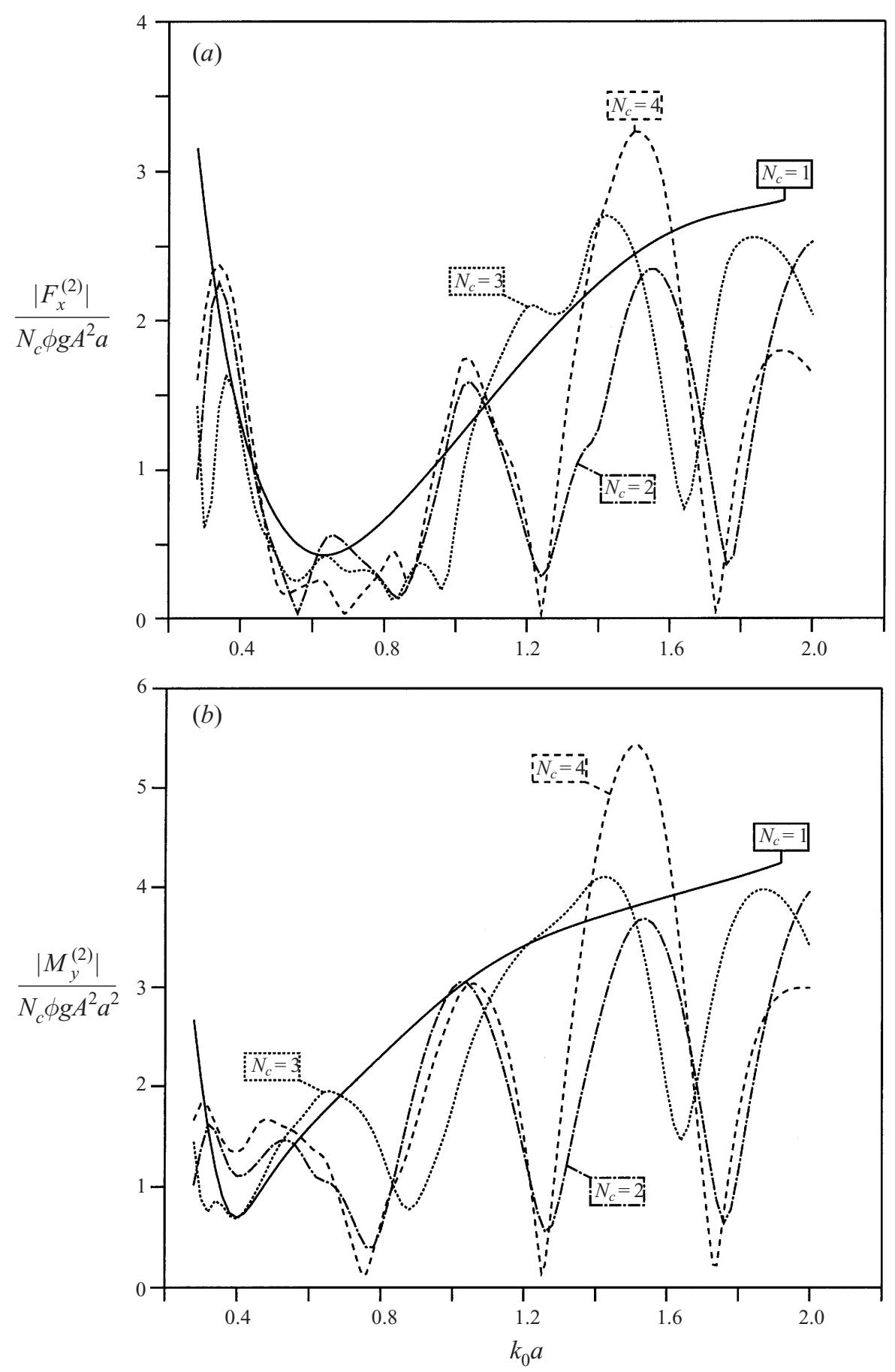

FIGURE 10. Averaged amplitude of $(a)$ the dimensionless second-order surge force and (b) the pitch moment for different cylinder configurations. Wave incidence is $\beta=0$. 

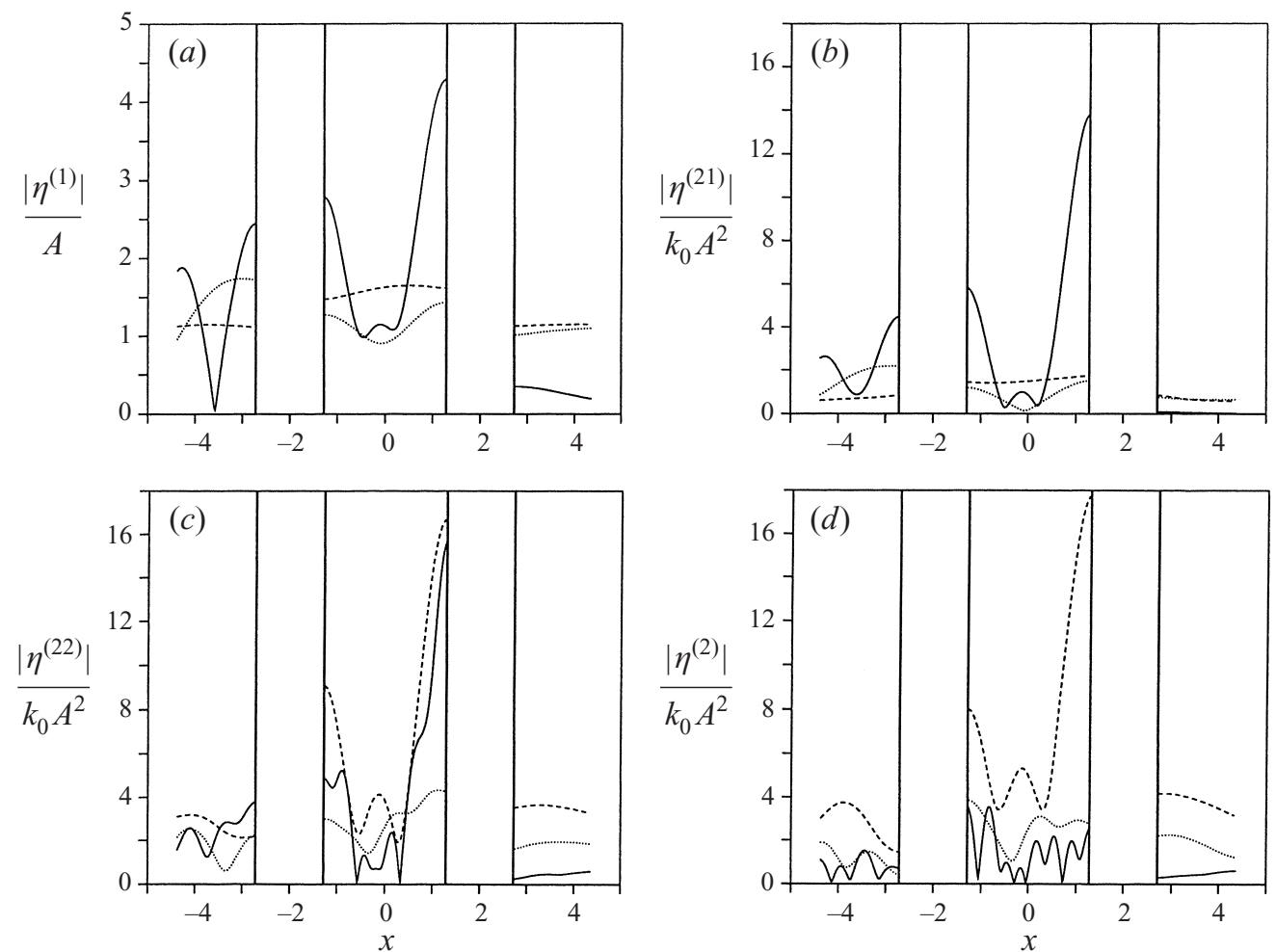

FIGURE 11. Components of non-dimensional first- and second-order wave elevation amplitude along $x=y$ for a four-cylinder array $(a / d=0.5)$ at near-trapping. Wave incidence is $\beta=\pi / 4$. Solid line: $k_{0} a=1.66$; dashed line: $k_{0} a=0.468$; dotted line: $k_{0} a=0.754$.

are associated respectively with quadratic products of the first-order quantities and with the second-order potential, as defined in equation (2.7). The total second-order elevation amplitude $\left|\eta^{(2)}\right|$ is given in figure 11(d). As found by Evans \& Porter (1997), $\left|\eta^{(1)}\right| / A$ is rather large $(>4)$ on the upwave face of cylinder 1 when $k_{0} a=1.66$, and elsewhere the incident wave amplitude is significantly increased. They have shown that this wavenumber corresponds to the phenomenon of a near-trapping mode in this configuration. Likewise, we see from the solid line in figure 11(b) that the secondorder component $\left|\eta^{(21)}\right|$ is also large at this position for $k_{0} a=1.66$, as expected. The amplitude of $\eta^{(22)}$ is similarly large, but its phase relative to $\eta^{(21)}$ is such that the total second-order elevation at this position is not especially large at this neartrapping frequency. Now consider the behaviour at $k_{0} a=0.468$ (the dashed line), which corresponds to one half of the near-trapping frequency in this water depth. We find that $\left|\eta^{(1)}\right|$ and $\left|\eta^{(21)}\right|$ are unremarkable, whereas $\left|\eta^{(22)}\right|$ is again large at the upper face of cylinder 1 . This highly significant result suggests that near-trapping of the second-order wave occurs when its frequency coincides with the linear near-trapping frequency. The magnitude of the effect is such that it dominates the total second-order wave (the dashed line shown in figure 11d). The results shown as dotted lines in these figures, however, show no such remarkable features. These being associated with $k_{0} a=0.754$ correspond to a wave frequency which is two thirds of the near-trapping frequency, so large magnification effects would not be anticipated at first or second order. 

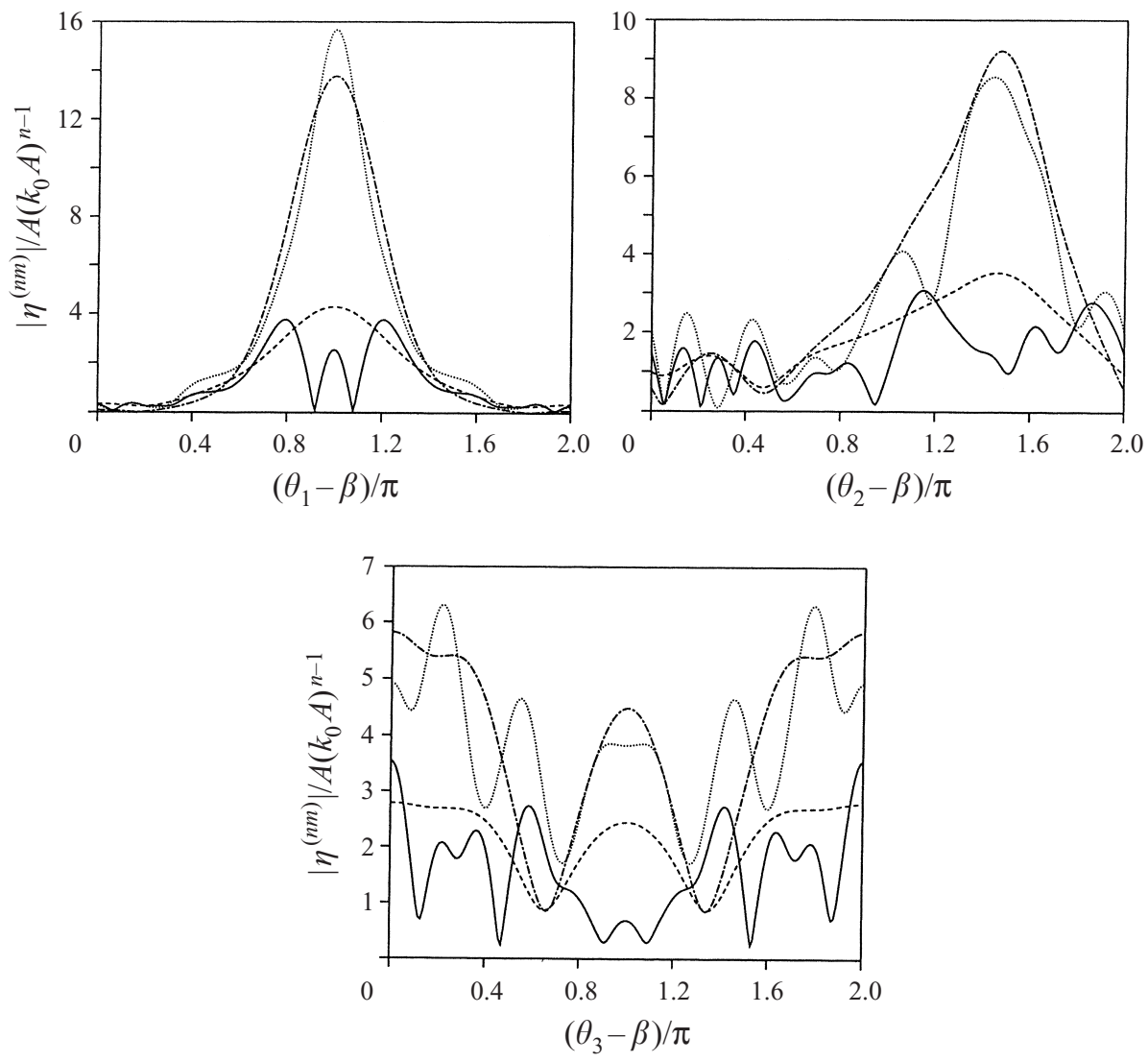

FIGURE 12. Components of non-dimensional first- and second-order wave run-up amplitude around cylinders in the array $(a / d=0.5)$ at $k_{0} a=1.66$. Wave incidence is $\beta=\pi / 4$. Dashed line: $\left|\eta^{(1)}\right| / A$; dot-dashed line: $\left|\eta^{(21)}\right| / k_{0} A^{2}$; dotted line: $\left|\eta^{(22)}\right| / k_{0} A^{2}$; full line: $\left|\eta^{(2)}\right| / k_{0} A^{2}$.

The influence of the near-trapping phenomenon on the first- and second-order waves is shown further in figures 12 and 13 . These show the components of first- and second-order run-up amplitude around three of the four cylinders (numbered anticlockwise from the cylinder in the positive quadrant, as for the results in figures 7 to 9). Figure 12 corresponds to the near-trapping frequency, i.e. with $k_{0} a=1.66$. One can observe the large first-order amplitudes of run-up at the inside faces of the cylinders, as also found by Evans \& Porter. The individual second-order components $\eta^{(21)}$ and $\eta^{(22)}$ are also large at these locations but, as noted above, due to their phase differences the associated total second-order elevation $\eta^{(2)}$ is relatively small for cylinders 1,2 and 4 . Figure 13 corresponds to half the near-trapping frequency, i.e. with $k_{0} a=0.468$. The linear run-up around all of the cylinders is seen to be small, whereas the second-order run-up is now large. The highest second-order run-up is at the inside face of cylinder 1 (the single downwave cylinder for this $\pi / 4$ wave incidence). This appears to correspond to a near-trapping phenomenon associated with the second-order wave.

Next we consider an arrangement where the cylinders are more closely spaced, at the corners of a square of side length $3.333 a(a / d=0.6)$. This case was also investigated by Evans \& Porter (1997). Figure 14 shows results analogous to those shown in figure 11 for $a / d=0.5$, but at a different set of frequencies: $k_{0} a=2.271$ 

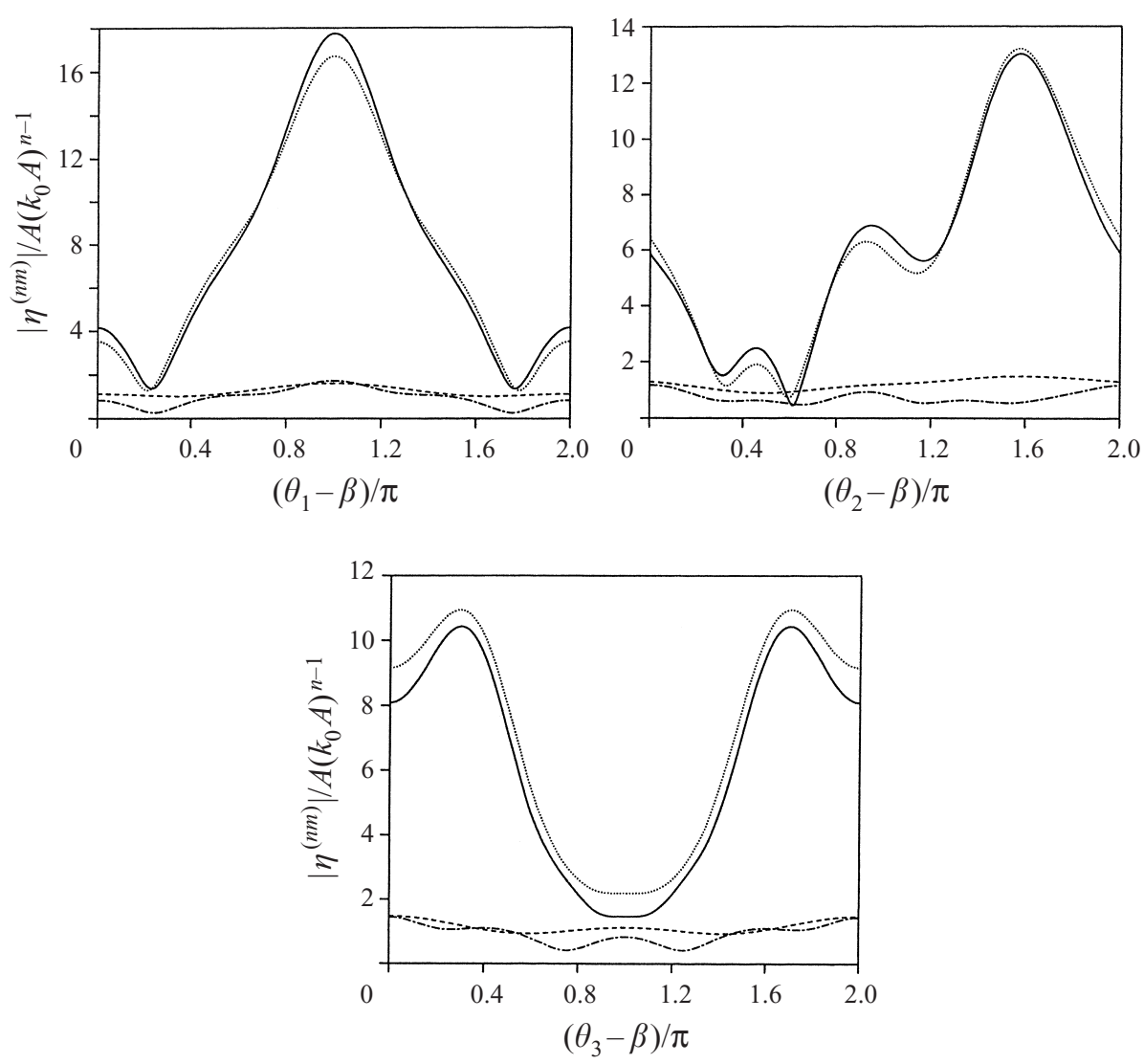

FIGURE 13 . As figure 12 but at $k_{0} a=0.468$.

corresponds to the near-trapping frequency in the linear problem, as shown by Evans $\&$ Porter, and $k_{0} a=0.600$ corresponds to half that frequency. The third set of results, for $k_{0} a=1.014$, corresponds to two thirds of the near-trapping frequency for this spacing. The behaviour is similar to that shown in figure 11, with large wave elevations occurring at the near-trapping frequency and at half that frequency. In this case with the cylinders now more closely spaced, the increases in the second-order amplitude are very much more pronounced (a factor of 40 for the elevation on the upwave face of the downwave cylinder). We can note again that at the first-order near-trapping frequency, $k_{0} a=2.271$ in this case, the components $\eta^{(21)}$ and $\eta^{(22)}$ largely cancel each other, so that the total second-order elevation amplitude $\left|\eta^{(2)}\right|$ at this frequency is not especially large. At half this near-trapping frequency, however (i.e. for $k_{0} a=0.600$ ), the total second-order amplitude $\left|\eta^{(2)}\right|$ is very large, because again $\left|\eta^{(22)}\right|$ is large but $\left|\eta^{(21)}\right|$ is not. This is further evidence suggesting that near-trapping of the second-order wave occurs when its frequency coincides with the linear near-trapping frequency.

The frequency (or wavenumber) dependence of the components of elevation may be seen more clearly in figure 15 . This corresponds to the four cylinders at the closer spacing $(a / d=0.6)$, and shows the run-up at the upwave face of the downwave cylinder. It is clear that the above-mentioned large increases in wave elevation only occur over a very narrow range of frequencies, close to what we might now describe as firstand second-order near-trapping. The peak in the second-order run-up corresponding 

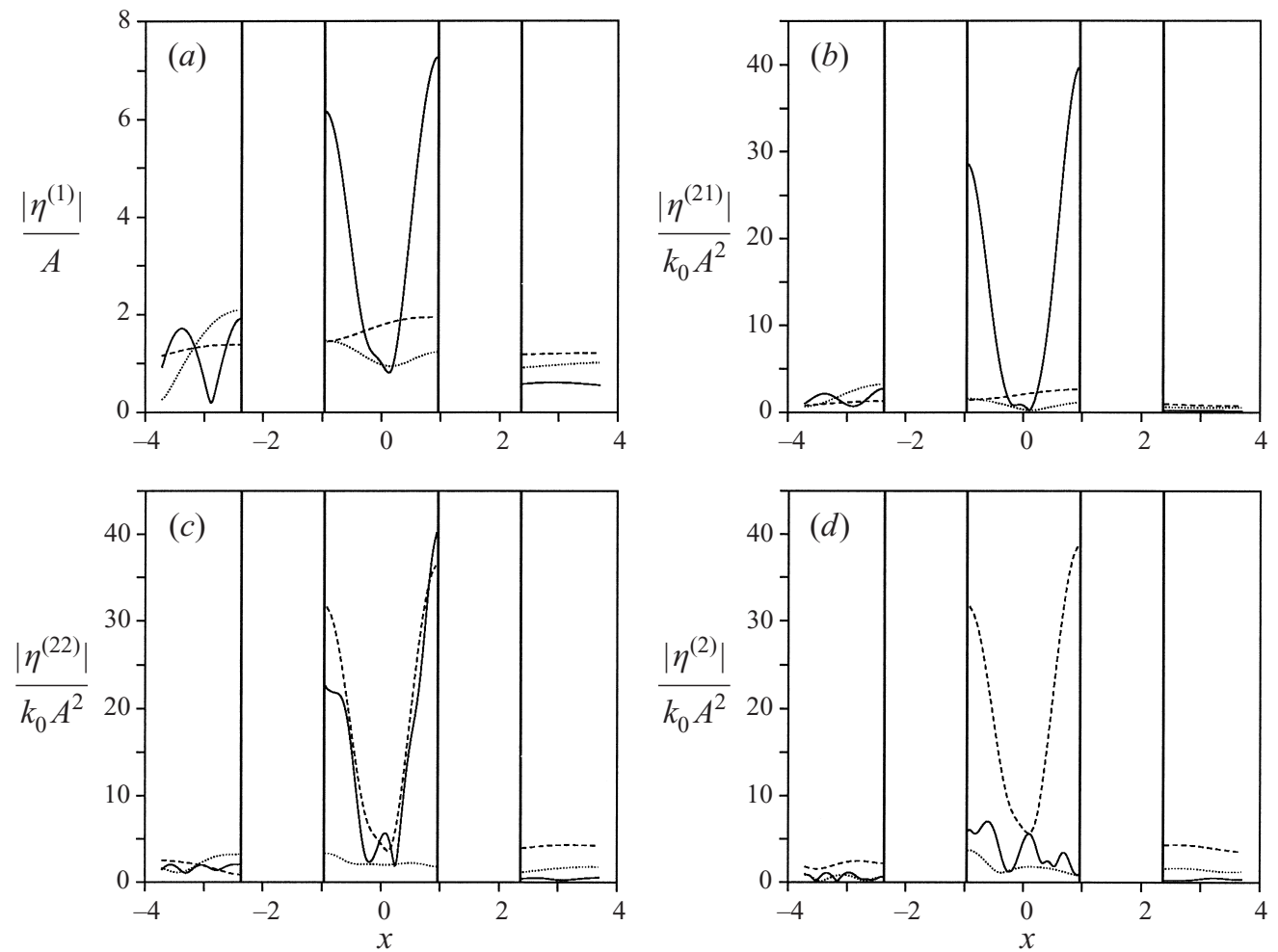

FIGURE 14. Components of non-dimensional first- and second-order wave elevation amplitude along $x=y$ for a four-cylinder array $(a / d=0.6)$ at near-trapping. Wave incidence is $\beta=\pi / 4$. Solid line: $k_{0} a=2.271$; dashed line: $k_{0} a=0.600$; dotted line: $k_{0} a=1.101$.

to second-order near-trapping (i.e. around $k_{0} a=0.600$ ) is even sharper than the peak in the first-order run-up at first-order near-trapping (i.e. around $k_{0} a=2.271$ ). The cancellation of the second-order components $\eta^{(21)}$ and $\eta^{(22)}$ at the first-order near-trapping frequency is also highlighted in figures $15(b)$ and $15(c)$ for the real and imaginary parts respectively.

All these results confirm the importance of the influence of interaction between cylinders on the final results. The idealization of an array of cylinders as a group of isolated cylinders is not justified and is wrong.

\section{Concluding remarks}

A complete semi-analytical solution for the second-order diffraction of an incident monochromatic wave by an array of vertical circular cylinders has been presented. As in the first-order case considered by Linton \& Evans (1990) and Evans \& Porter (1997), the importance of interaction effects when calculating the local or global quantities is again highlighted. The empirical evidence of the results shown in this paper suggests that the phenomenon of near-trapping in cylinder arrays, identified by Evans \& Porter for the linear wave problem, also occurs at second order. In particular, near-trapping has been shown to occur when the frequency of the second-order wave coincides with the linear near-trapping frequency. The resulting magnification of the local wave elevation near the cylinders can be extremely high. 

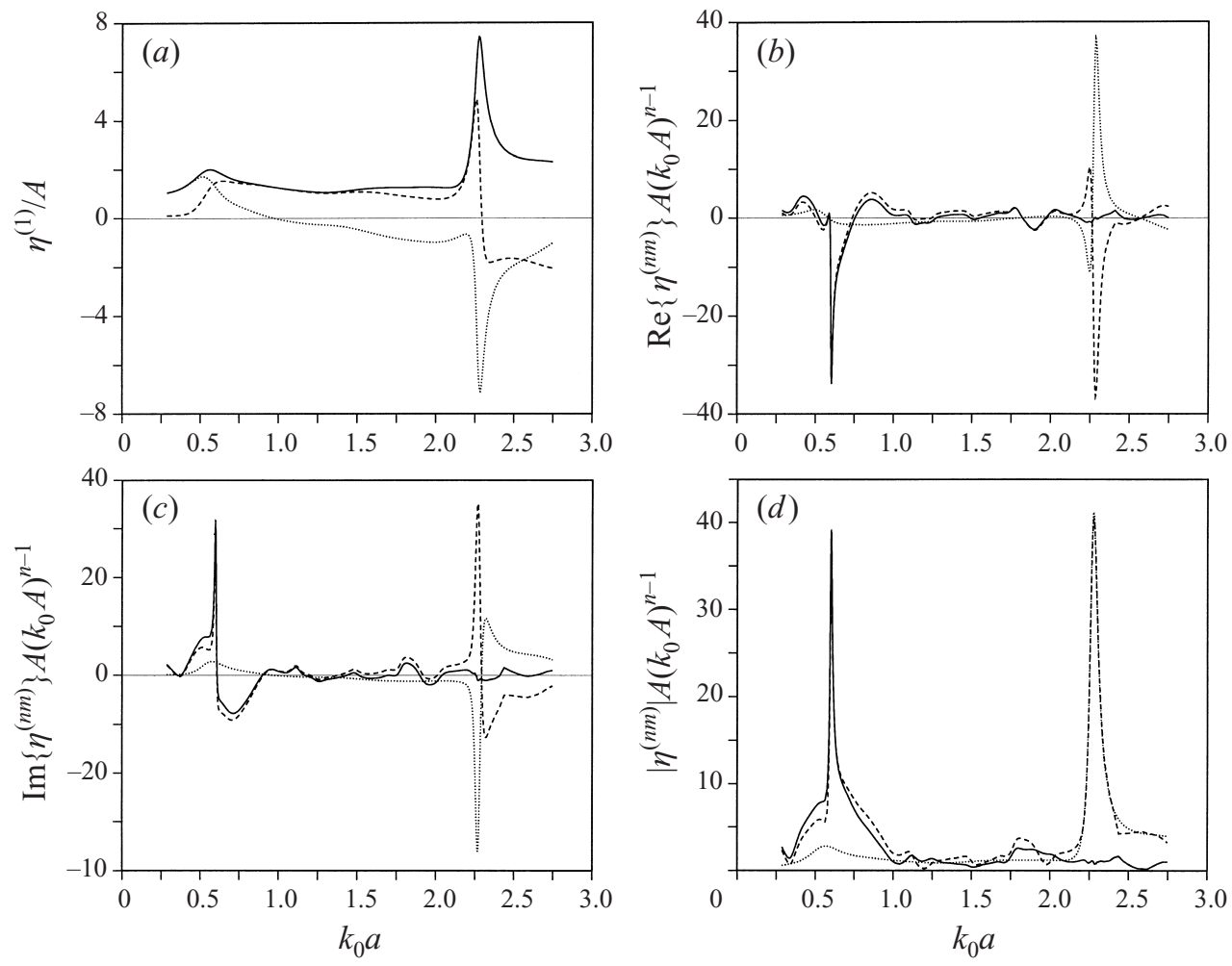

FigURE 15. Frequency dependence of dimensionless run-up in the array $(a / d=0.6)$. Wave incidence is $\beta=\pi / 4$. (a) First-order component. Solid line: magnitude; dashed line: real part; dotted line: imaginary part. (b) Real parts of second-order components. (c) Imaginary parts of second-order components. (d) Magnitudes of second-order components. In $(b-d)$ solid line: $\eta^{(2)} / k_{0} A^{2}$; dotted line: $\eta^{(21)} / k_{0} A^{2}$; dashed line: $\eta^{(22)} / k_{0} A^{2}$.

The next step in the analysis should be extension to the evaluation of thirdorder forces. This can be done by the indirect approach referred to above, using the Haskind relations, provided that the second-order potential and its derivatives can be accurately evaluated on a very large part of the free surface. This paper has provided the basis for evaluating the potential to the required level of accuracy. The remaining difficulty associated with the third-order calculation is the evaluation of the free surface term involving second-order derivatives of the second-order potential, especially in the intermediate region (i.e. as shown in figure 2).

Lastly we remark that adopting a semi-analytical approach for the case of multiple cylinders can provide important insights into the behaviour of practical platforms such as TLPs. But it should also be noted that, even with this approach, the formulation is complex, and the CPU time required is low only if the programming is carried out carefully.

The first author carried out this work within the scope of the CLAROM project 'Large amplitude wave amplifications in the vicinity of offshore structures' in which the partners are Bureau Veritas, Bouygues Offshore, Doris Engineering, GESIM, Principia R \& D and SIREHNA. The other authors acknowledge the support of grant GR/L19355 from the UK Engineering and Physical Sciences Research Council. 


\section{REFERENCES}

Abramowitz, M. \& Stegun, I. 1970 Handbook of Mathematical Functions. Dover.

CHAU, F. P. 1989 The second-order velocity potential for diffraction of waves by fixed offshore structures. PhD thesis, University of London.

Chau, F. P. \& EATOCK TAYlor, R. 1992 Second order wave diffraction by a vertical cylinder. $J$. Fluid Mech. 240, 571-599.

Chen, X. B. 1991 Second-order high frequency loads on tension leg platform columns. Institut Francais du Petrole, Rep. 38741.

Chen, X. B., Molin, B. \& Petitjean, F. 1991 Faster evaluation of resonant exciting loads on tension leg platform. In Proc. VII Intl Symp. Offshore Engng, Brasil Offshore '91, Rio de Janeiro.

Chen, X. B., Molin, B. \& Petitjean, F. 1995 Numerical evaluation of the springing loads on tension leg platforms. Marine Structures 8, 501-524.

Eatock Taylor, R. \& Chau, F. P. 1992 Wave diffraction theory. Some developments in linear and nonlinear theory. J. Offshore Mech. Arctic Engng 114, 185-194.

EATOCK TAYlor, R. \& Huang, J. B. 1997a Second-order wave diffraction by an axisymmetric body in monochromatic waves. Proc. R. Soc. Lond. A 453, 1515-1541.

EAtock Taylor, R. \& Huang, J. B. 1997b Semi-analytical solution for second-order diffraction by a vertical cylinder in bichromatic waves. J. Fluids Structures 11, 465-484

Evans, D. V. \& PORTER, R. 1997 Near trapping of waves by circular arrays of vertical cylinders. Appl. Ocean Res. 19, 83-99.

Fenton, J. D. 1978 Wave forces on vertical bodies of revolution. J. Fluid Mech. 85, 241-255.

FERrANT, P. 1996 Simulation of strongly non-linear wave generation and wave-body interactions using a three-dimensional MEL model. In Proc. 21st Sympos. on Naval Hydrodynamics, Trondheim, Norway.

Huang, J. B. \& EAtock TaYlor, R. 1996a Semi-analytical solution for second-order wave diffraction by a truncated circular cylinder in monochromatic waves. J. Fluid Mech. 319, 171-196.

Huang, J. B. \& EATOCK TAYLOR, R. $1996 b$ Second-order interaction between waves and multiple bottom-mounted vertical circular cylinders. In 11th Intl Workshop on Water Waves and Floating Bodies, Hamburg, Germany (ed. V. Bertram). Institut für Schiffbau.

Kim, M. H. \& YuE, D. K. P. 1989 The complete second-order diffraction solution for an axisymetric body. Part 1. Monochromatic incident waves. J. Fluid Mech. 200, 235-264.

KRIEBEL, D. L. 1990 Nonlinear wave interaction with a vertical circular cylinder, part I: diffraction theory. Ocean Engng 17, 345-377.

Lighthill, M. J. 1979 Waves and hydrodynamic loading. In Proc. 2nd Intl Conf. on Behaviour of Offshore Structures, BOSS 79, London, UK (ed. H. S. Stephens \& B. Knight), vol. 1, pp. 1-40.

Linton, C. M. \& Evans, D. V. 1990 The interaction of waves with arrays of vertical circular cylinders. J. Fluid Mech. 215, 549-569.

MALENICA, Š. 1997 Second-order interaction of water waves with arrays of vertical cylinders. In Proc. 2nd Congress of Croatian Society of Mechanics, Supetar, Croatia, pp. 599-606.

Malenica, Š. \& Molin, B. 1995 Third harmonic wave diffraction by a vertical cylinder. J. Fluid Mech. 302, 203-229.

Molin, B. 1979 Second-order diffraction loads upon three-dimensional bodies. Appl. Ocean Res. 1, 197-202.

Molin, B. 1994 Second-order hydrodynamics applied to moored structures. Ship Tech. Res. 41/2.

Newman, J. N. 1990 Second harmonic wave diffraction at large depths. J. Fluid Mech. 213, 61-98.

Newman, J. N. 1996 The second-order wave force on a vertical cylinder. J. Fluid Mech. 320, 417-443.

Newman, J. N. \& Lee, C. H. 1992 Sensitivity of wave loads to the discretisation of bodies. In Proc. 6th Intl Conf. on Behaviour of Offshore Structures, BOSS 92, London, UK (ed. M. H. Patel \& R. Gibbins), vol. 1, pp. 50-64.

Romate, J. E. 1989 The numerical simulation of nonlinear gravity waves in three dimensions using a higher order panel method. PhD thesis, University of Twente, The Netherlands.

SCOLAN, Y. M. \& MALENICA, Š. 1998 Experimental and numerical second-order diffracted waves around an array of four cylinders. In Proc. 13th Intl Workshop on Water Waves and Floating Bodies, Alphen aan den Rijn, The Netherlands (ed. A. J. Hermans), pp. 135-138. Delft University of Technology. 
Scolan, Y. M. \& Molin, B. 1989 Second-order deformation of the free surface around a vertical cylinder. In Proc. 4th Intl Workshop on Water Waves and Floating Bodies, Oystese, Norway (ed. J. Grue), pp. 227-232. University of Oslo.

Xue, M. \& YuE, D. K. P. 1995 Fully nonlinear three dimensional interaction between water waves and a surface piercing body. In Proc. 10th Intl Workshop on Water Waves and Floating Bodies, Oxford, UK (ed. R. Eatock Taylor), pp. 262-266. University of Oxford. 